\title{
Numerical study for soil response around submerged breakwaters with Bragg reflection
}

\author{
L Cui \\ School of Engineering \& Built Environment, Griffith University \\ Gold Coast Campus, Queensland, 4222, Australia \\ lin.cui3@griffithuni.edu.au \\ D-S Jeng \\ School of Engineering $\& 3$ Built Environment, Griffith University \\ Gold Coast Campus, Queensland, 4222, Australia \\ d.jeng@griffith.edu.au \\ Received (Day Month Year) \\ Revised (Day Month Year)
}

\begin{abstract}
A better understanding of soil behaviour in a seabed foundation around submerged breakwaters under combined wave and current loadings has become crucial regarding the design and maintenance for such breakwaters. Bragg effect is considered in this study, which is one of important factors that influence the flow field and soil response in the vicinity of multiple breakwaters. The wave-current induced dynamic soil response (effective stresses, pore pressures and displacements) and its resultant residual liquefaction in a loosely deposited seabed foundation around multiple breakwaters are investigated. In this study, the wave motion is governed by VARANS equation and the Biot's $u-p$ approximation is used to govern soil-fluid interactions in porous medium. The elasto-plastic constitutive model (PZIII) is used to reproduce the plastic soil behaviour in seabed foundation under long-term cyclic ocean loading. Numerical results show that the flow motion can be largely changed due to Bragg effects. The construction of breakwaters significantly change the stress field in seabed foundation. Parametric study shows that, under the strongest Bragg effect, the presence of currents, soil properties and wave characteristics have great impact on the liquefaction potential.
\end{abstract}

Keywords: VARANS; $u-p$ approximation; Bragg effect; multiple submerged permeable breakwaters; loosely deposited soil; wave-current interaction.

\section{Introduction}

Submerged breakwaters have been used by coastal engineers because of the following advantages: (1) less impact on the ecological environment as crown of the breakwater is under water surface; and (2) the ability to reflect and dissipate wave energy efficiently due to the friction effect within the porous medium. Owing to its unique advantages, submerged breakwaters have been widely used to protect coastlines as an offshore structure. Stability of submerged breakwater under natural environmen- 
tal loadings in ocean environments has become one of the main issues involving in design and maintenance of the structures. As reported in the literature, numerous damages of submerged breakwaters were caused by wave-induced seabed instability rather than from the construction deficiencies [Sumer, 2009]. Therefore, it is essential to have a better understanding of the seabed response around breakwaters under the ocean environmental loading.

There are numerous factors that influence the flow field and soil response around submerged breakwaters. One of them is Bragg effect, which is defined as amplification of reflected waves by multiple breakwaters. This normally occurs when the spaces between two adjacent breakwaters are about half of incident wavelength. This effect is formed by the constructive interference of incident and reflected waves from successive bottom undulation, which is well known in x-ray diffraction by crystalline materials [Mei et al., 2005]. Bragg effect can change the wave motion and its resultant soil response around breakwaters. On the one hand, Bragg effect is beneficial for breakwaters to protect the coastlines as more waves will be reflected. On the other hand, a seabed foundation is more likely to be liquefied as the wave height become larger at the seaward side of the breakwaters. Most previous investigations have focused on single submerged permeable breakwater without considering Bragg reflection. To authors' best knowledge, only Zhang et al. [2012] studied the seabed response and liquefaction potential around multiple submerged permeable breakwaters subject to Bragg reflection. However, their model was limited to poro-elastic seabed model, which is only valid for small strain. The poro-elastoplastic soil behaviours such as permanent deformation under long-term cyclic wave loading are not able to be determined by using such a model. As the result, including a more advanced elasto-plastic constitutive model into the seabed model is desired.

In natural ocean environments, waves and currents simultaneously exist and always interact with each other. The presence of currents could significantly change the hydrodynamic properties in flow field and further affect the dynamic response within a porous seabed. However, most previous investigations of soil response around offshore structures focused on the wave loading only without considering the effects of current. Recently, some works were carried out. Among these, Ye and Jeng [2012] were the first including current in their model to study seabed response and liquefaction. In their work, the third-order approximation of non-linear wave-current interaction was used to determine the dynamic pressure acting on the surface of seabed foundation in soil model. However, their study didn't consider any structure at all.

In the past few decades, considerable studies have been carried out to investigate the problems of wave-structures-seabed interactions [Jeng et al., 2005]. In the early stage, analytical solutions are the most widely used method to study the wave induced soil response around submerged permeable breakwater, for example, Jeng [1996] treated the breakwaters as lines without width and weight. Later, numerous numerical models are developed to study the wave-breakwater-seabed interactions. 
These models normally consist of two sub-models: fluid model for fluid-structure interactions and seabed model for dynamic soil response. The problem of fluidstructure interactions has been intensively studied previously [Lin and Liu, 1998; Liu et al., 1999; Hsu et al., 2002]. In these studies, the Navier-Stokes equation has been used to reproduce local hydrodynamic process with interactions with offshore structures. These flow model could be used to predict free water surface, velocity field and hydrodynamic pressure in the flow field. The computational results from flow model could be used as boundary conditions in the seabed model. By integrating fluid and seabed models, the integrated numerical model could simulate the more realistic engineering problems as the effect of structure shape, porous properties and the gravity of structures are be included.

The aforementioned investigations mainly focused on poro-elastic behaviours of seabed foundation, which are limited to relative dense soil with small strain under dynamic wave loading. However, in the case of soil with large deformation, for example, loosely deposited sandy soil, the poro-elastic model may be insufficient. Therefore, it is necessary to include a more advanced elasto-plastic constitutive model in the seabed model with better capability to describe the non-linear behaviour of loosely deposited soil. In this study, poro-elastoplastic constitutive model Pastor-Zienkiewicz Mark-III (PZIII) is adopted, which was proposed by Zienkiewicz and Mroz [1984], Chan [1988] and Pastor et al. [1990]. The original PZIII model was developed for earthquake loadings. Based on PZIII, Dunn et al. [2006] numerically studied the wave-induced liquefaction in loose seabed around buried pipelines under progressive wave action. Later, Jeng and Ou [2010] further extended PZIII model from $2 \mathrm{D}$ to $3 \mathrm{D}$ to investigate the wave induced pore pressure in loose seabed near breakwater heads. More recently, Ye et al. [2015] adopted PZIII constitutive model in their integrated numerical model FSSI-CAS 2D to investigate the wave induced dynamic response of a composite breakwater and the seabed foundation.

In this paper, an integrated numerical model is developed to investigate dynamic soil response and liquefaction potential in an elasto-plastic seabed foundation around multiple breakwaters under combined wave and current loadings and Bragg reflection. The flow model is simulated by IHFOAM [Higuera et al., 2013] and the numerical code for seabed model is based on DIANA-SWANDYNE II [Chan, 1988; Dunn et al., 2006]. The model is verified with published experimental data first. Then, the wave-current-structures interactions will be studied to find the strongest Bragg reflection. To simulate the realistic nature condition, a new balance state after process of consolidation due to the presence of multiple breakwaters will be determined first and used as the initial conditions for later dynamic soil analysis. Finally, the dynamic soil response and liquefaction potential in an elasto-plastic seabed foundation will be examined. The parametric studies will be further carried out to examine the effects of current, soil properties and wave characteristics on wave-current induced liquefaction potential. 


\section{Numerical Model}

In this study, an integrated model, consisting of flow and seabed sub-models, is used to simulate fluid-structures-seabed interactions. The flow sub-model employed for investigating wave-current-breakwaters interactions is developed using open-source CFD toolbox OpenFOAM ${ }^{\circledR}$ (Open Field Operation and Manipulation). The seabed sub-model is adopted to describe the nonlinear plastic behaviour of seabed foundation, which is based on finite element program DIANA-SWANDYNE II (Dynamic Interaction And Nonlinear Analysis-SWANsea DYNamic version II) [Chan, 1988; Dunn et al., 2006; Jeng and Ou, 2010].

\subsection{Flow sub-model}

The flow sub-model is based on one of the solvers in OpenFOAM ${ }^{\circledR}$, IHFOAM, which is designed to simulate coastal, offshore and hydraulic engineering processes. IHFOAM solves the three-dimensional Volume Averaged Reynolds Averaged NavierStokes (VARANS) equations for two incompressible phases (water and air) using a finite volume discretization and volume of fluid (VOF) method [Higuera et al., 2013]. The governing equations including the mass conservation and momentum conservation equations can be expressed as:

$$
\begin{gathered}
\frac{\partial\left\langle u_{i}\right\rangle}{\partial x_{i}}=0 \\
\frac{\partial \rho\left\langle u_{i}\right\rangle}{\partial t}+\frac{\partial}{\partial x_{j}}\left[\frac{1}{n} \rho\left\langle u_{i}\right\rangle\left\langle u_{j}\right\rangle\right]=-n \frac{\partial\left\langle p^{*}\right\rangle^{f}}{\partial x_{i}}+n \rho g_{i}+\frac{\partial}{\partial x_{j}}\left[\mu_{e f f} \frac{\partial\left\langle u_{i}\right\rangle}{\partial x_{j}}\right]-[C T],
\end{gathered}
$$

where \langle\rangle and \langle\rangle$^{f}$ are Darcy's volume averaging operator and the intrinsic averaging operator, respectively; $\rho$ is the density, computed by $\rho=\alpha \rho_{\text {water }}+(1-\alpha) \rho_{\text {air }}$, in which $\alpha$ is the indicator function defined in Equation (5); $\mathbf{U}, u_{i}$ is the velocity vector; $n$ is the porosity; $p^{*}$ is the pseudo-dynamic pressures; $\mathbf{g}, g_{i}$ is the gravitational acceleration; $\mu_{e f f}$ is the efficient dynamic viscosity, defined as $\mu_{\text {eff }}=\mu+\rho \nu_{\text {turb }}$, in which $\mu$ is the molecular dynamic viscosity and $\nu_{\text {turb }}$ is the turbulent kinetic viscosity, given by the chosen turbulence model. In this study, the $k-\epsilon$ turbulence model is used; the last term in Equation (2) represents the resistance of porous media, can be expressed as:

$$
[C T]=A\left\langle u_{i}\right\rangle+B|\langle u\rangle|\left\langle u_{i}\right\rangle+C \frac{\partial\left\langle u_{i}\right\rangle}{\partial t},
$$

where the factor $C$ is less significant to factors $A$ and $B$ and a value of $C=0.34$ $\left[\mathrm{kg} / \mathrm{m}^{3}\right]$ is often applied by default [del Jesus, 2011].

Regarding values of $A$ and $B$, Engelund [1953]'s formulae, as applied in Burcharth and Andersen [1995], are employed in the present model to determine the resistance coefficients as:

$$
A=E_{1} \frac{(1-n)^{3}}{n^{2}} \frac{\mu}{D_{50}^{2}}, \text { and } B=E_{2}\left(1+\frac{7.5}{K C}\right) \frac{1-n}{n^{2}} \frac{\rho}{D_{50}},
$$


in which $D_{50}$ is the medium grain diameter of the materials; $K C$ is the Keulegan Carpenter number, which is defined as $K C=\frac{u_{m} T}{n D_{50}}$, in which $u_{m}$ is the maximum oscillating velocity and $T$ is the period of the oscillation. $E_{1}$ and $E_{2}$ are parameters that characterize the linear and non-linear friction terms. A sensitivity analysis of these parameters on the solutions for present study has been carried out and the numerical results are given in Appendix. The results show that the hydrodynamic pressure is not sensitive to the variation of these parameters. Therefore, the default values of these parameters $\left(E_{1}=50\right.$ and $\left.E_{2}=1.2\right)$ are used in this study [Higuera, 2015].

Each cell in the computational domain is considered as a mixture of two-phase fluid (air and water). The indicator function $\alpha$ varies from 0 (air) to 1 (water), defined as the quantity of water per unit of volume at each cell, which is shown as:

$$
\alpha=\left\{\begin{array}{lc}
1, & \text { water } \\
0, & \text { air } \\
0<\alpha<1, & \text { free surface }
\end{array}\right.
$$

Any spatial variation of fluid properties, such as density and viscosity, can be represented using the indicator function $\alpha$ considering the mixture properties:

$$
\Phi=\alpha \Phi_{\text {water }}+(1-\alpha) \Phi_{\text {air }},
$$

where $\Phi_{\text {water }}$ and $\Phi_{\text {air }}$ are the properties of water and air respectively.

The fluid movement could be tracked by solving the advection equation as:

$$
\frac{\partial \alpha}{\partial t}+\frac{1}{n} \frac{\partial\left\langle u_{i}\right\rangle \alpha}{\partial x_{i}}+\frac{1}{n} \frac{\partial\left\langle u_{c i}\right\rangle \alpha(1-\alpha)}{\partial x_{i}}=0
$$

where $\left|\mathbf{U}_{c}\right|=\min \left[c_{\alpha}|\mathbf{U}|, \max (|\mathbf{U}|)\right]$, in which the default value of $c_{\alpha}$ is 1 , however, the user can specify a greater value to enhance the compression of the interface, or zero to eliminate it.

The solving algorithm used in flow model is PIMPLE, which is a combination of PISO (Pressure Implicit with Splitting of Operators) and SIMPLE (Semi-Implicit Method for Pressure-Linked Equations) algorithms. In this study, the $k-\epsilon$ RAS (Reynolds-averaged simulation) turbulence model is adopted to model the turbulent viscosity $\nu_{\text {turb }}$ as:

$$
\nu_{\text {turb }}=\frac{C_{\mu} k^{2}}{\epsilon}
$$

where $C_{\mu}$ is an empirical constant; $k$ and $\epsilon$ are the turbulence kinetic energy and the turbulence energy dissipation rate, respectively.

The wave generation and active wave absorption in the fluid domain are implemented within the IHFOAM. Several boundary conditions are introduced: (i) the inlet boundary condition allow the generating of wave according to different wave theories as well as adding different steady current flow; (ii) the outlet boundary condition applies an active wave absorption theory to prevent the re-reflection of 
incoming wave; (iii) the slip boundary condition (zero-gradient) is applied on the bottom of the fluid domain and the lateral boundary of the numerical wave flume; (iv) the top boundary condition is set as the atmospheric pressure. For the details of IHFOAM toolbox, the readers can refer to Higuera et al. [2013].

\subsection{Seabed sub-model}

\subsubsection{Governing equation: $u-p$ approximation}

A porous seabed is formed by solid particles with voids filled by pore water and/or air distributed through the body. In this study, the $u-p$ approximation proposed by Zienkiewicz et al. [1980] is used as the governing equations to govern the interactions between solid skeleton and pore fluid in a porous seabed, in which the relative displacements of pore fluid to soil particles are ignored and the acceleration of pore fluid and solid particles is included. The $u-p$ approximation equations can be expressed in conjunction with Equations (9) to (11). Note that these equations are for two dimensional situations. Since this study focuses on two dimensional cases, only $2 \mathrm{D}$ equations are presented. For the $u-p$ approximation equations in threedimensional cases, readers can refer to Jeng and Ou [2010].

$$
\begin{gathered}
\frac{\partial \sigma_{x}^{\prime}}{\partial x}+\frac{\partial \tau_{x z}}{\partial z}=-\frac{\partial p_{s}}{\partial x}+\rho \frac{\partial^{2} u_{s}}{\partial t^{2}}, \\
\frac{\partial \tau_{x z}}{\partial x}+\frac{\partial \sigma_{z}^{\prime}}{\partial z}+\rho g=-\frac{\partial p_{s}}{\partial z}+\rho \frac{\partial^{2} w_{s}}{\partial t^{2}}, \\
k \nabla^{2} p_{s}-\gamma_{w} n_{s} \beta \frac{\partial p_{s}}{\partial t}+k \rho_{f} \frac{\partial^{2} \epsilon_{s}}{\partial t^{2}}=\gamma_{w} \frac{\partial \epsilon_{s}}{\partial t} .
\end{gathered}
$$

where $\sigma_{x}^{\prime}$ and $\sigma_{z}^{\prime}$ are the effective stresses in $x$ - and $z$ - directions, respectively; $p_{s}$ is the pore pressure; $u_{s}$ and $w_{s}$ are soil displacements in $x$ - and $z$-directions, respectively; $\tau_{x z}$ is the shear stress; $\rho=\rho_{f} n_{s}+\rho_{s}\left(1-n_{s}\right)$ is the average density of porous seabed, in which $\rho_{f}$ is fluid density and $\rho_{s}$ is soil density; $g$ is the gravitational acceleration; $k$ is Darcy's permeability; $\gamma_{w}$ is the unit weight of water; $\beta$ and $\epsilon_{s}$ are the compressibility of pore fluid and volume strain respectively, which are defined as below:

$$
\beta=\left(\frac{1}{K_{f}}+\frac{1-S_{r}}{p_{w 0}}\right), \text { and } \epsilon_{s}=\frac{\partial u_{s}}{\partial x}+\frac{\partial w_{s}}{\partial z},
$$

where $S_{r}$ is the degree of saturation; $p_{w 0}$ is the absolute static pressure and $K_{f}$ is bulk modulus of pore fluid, generally, $K_{f}=1.95 \times 10^{9} \mathrm{~N} / \mathrm{m}^{2}$ for pore water [Yamamoto et al., 1978].

\subsubsection{Constitutive model of a porous seabed: PZIII}

As mentioned previously, most previous investigations of wave-seabed-structure interactions have been limited to poro-elastic models [Jeng, 2003], which is valid for 
oscillatory soil responses. In this study, the advanced poro-elastoplastic constitutive model Pastor-Zienkiewicz Mark-III (PZIII) is adopted to describe the plastic soil behaviour under monotonic loading. The plasticity of soil obtained by classic elastoplastic model can be ignored when subject to small amplitudes cyclic loading like wave loading. This is because the load-unload cycle may belong to the yield surface interior where the elastic deformation takes place. The advanced PZIII model utilizes the generalized plasticity framework, in which no yield or plastic potential surface are explicitly defined [Pastor et al., 1990].

In the governing equations (9)-(11), the effective normal stresses can be obtained by multiplying the strains by elasto-plastic matrix $D^{e p}$ :

$$
D_{i j k l}^{e p}=D_{i j k l}^{e}-\frac{D_{i j m n}^{e} m_{m n} n_{s t} D_{s t k l}^{e}}{H_{L / U}+n_{s t} D_{s t k l}^{e} m_{k l}},
$$

where $D_{i j k l}^{e}$ is the tensor form of elastic matrix; $H_{L / U}$ is the plastic modulus at loading and unloading stages; $m_{m n}$ and $n_{s t}$ are the plastic flow direction tensor and the loading or unloading direction tensor, defined as:

$$
m_{m n}=\frac{\frac{\partial g}{\partial \sigma_{m n}^{\prime}}}{\left\|\frac{\partial g}{\partial \sigma^{\prime}}\right\|}, \quad \text { and } \quad n_{s t}=\frac{\frac{\partial f}{\partial \sigma_{s t}^{\prime}}}{\left\|\frac{\partial f}{\partial \sigma^{\prime}}\right\|},
$$

where $\left\|\partial g / \partial \sigma^{\prime}\right\|$ and $\left\|\partial f / \partial \sigma^{\prime}\right\|$ are the norm of the tensor $\partial g / \partial \sigma_{i j}^{\prime}$ and $\partial f / \partial \sigma_{i j}^{\prime}$, respectively; $g$ and $f$ are the plastic potential surface function and yield surface function in stress space, respectively. In this study, the non-associated flow rule is applied since the yield surface function $(f)$ and plastic potential surface function $(g)$ are different, which are define as:

$$
\begin{aligned}
& f=q^{\prime}-M_{f} p^{\prime}\left(1+\frac{1}{\alpha_{f}}\right)\left[1-\left(\frac{p^{\prime}}{p_{f}^{\prime}}\right)^{\alpha_{f}}\right]=0, \\
& g=q^{\prime}-M_{g} p^{\prime}\left(1+\frac{1}{\alpha_{g}}\right)\left[1-\left(\frac{p^{\prime}}{p_{g}^{\prime}}\right)^{\alpha_{g}}\right]=0,
\end{aligned}
$$

where $p_{f}^{\prime}$ and $p_{g}^{\prime}$ are constants characterizing the size of yield surface; $g$ and $f$ are plastic potential surface function and yield surface function defined in PZIII model [Pastor et al., 1990]; $q^{\prime}$ and $p^{\prime}$ are the generalised shear stress and mean effective stress, respectively, which can be determined as:

$$
\begin{gathered}
q^{\prime}=\left\{\frac{1}{3}\left(\sigma_{11}^{\prime}-\sigma_{22}^{\prime}\right)^{2}+\left(\sigma_{11}^{\prime}-\sigma_{33}^{\prime}\right)^{2}+\left(\sigma_{22}^{\prime}-\sigma_{33}^{\prime}\right)^{2}+6\left(\sigma_{12}^{2}+\sigma_{23}^{2}+\sigma_{31}^{2}\right)\right\}^{1 / 2}, \\
p^{\prime}=\frac{1}{3}\left(\sigma_{11}^{\prime}+\sigma_{22}^{\prime}+\sigma_{33}^{\prime}\right),
\end{gathered}
$$

The plastic modulus at loading and unloading stage are defined as:

$$
H_{L}=H_{0} p^{\prime}\left(1-\frac{q^{\prime} / p^{\prime}}{\eta_{f}}\right)^{4}\left[1-\frac{q^{\prime} / p^{\prime}}{M_{g}}+\beta_{0} \beta_{1} \exp \left(-\beta_{0} \xi\right)\right]\left(\frac{q / p^{\prime}}{\eta_{\max }}\right)^{-\gamma D M},
$$


8 L Cui \& D-S Jeng

$$
H_{U}=\left\{\begin{array}{lll}
H_{u 0}\left(\frac{M_{g}}{\eta_{u}}\right)^{\gamma U}, & \text { for } & \left|\frac{M_{g}}{\eta_{u}}\right|>1 \\
H_{u 0}, & \text { for } & \left|\frac{M_{g}}{\eta_{u}}\right| \leq 1
\end{array}\right.
$$

where $M_{f}, M_{g}, \alpha_{f}, \alpha_{g}, H_{0}, H_{u} 0, \eta_{u}, \eta_{\max }, \beta_{0}, \beta_{1}, \gamma_{u}$ and $\gamma_{D M}$ are the parameters describing the sandy soil. For the detailed definition of these parameters, readers can refer to [Pastor et al., 1990].

\subsection{Integration of flow and seabed sub-models}

For the integration of numerical models, a one-way coupling algorithm is adopted within two separate sub models. The detailed coupling process between the flow model and seabed model is illustrated in Figure 1. A data exchange port is developed between flow domain and solid domain. The flow model is responsible for generating and propagating of flow over the solid domain, as well as the porous flow inside porous medium according to the input of wave/current characteristics. The hydrodynamic properties including hydrodynamic pressure at the interface between fluid domain and solid domain could be obtained after solving the VARANS equations. The hydrodynamic pressure will be extracted from the results obtained from the flow model and applied to the seabed surface as the external loading for the seabed model through the data exchange interface between the VARANS equations and the $u-p$ approximation equations. Along with other input parameters of seabed model, the dynamic soil response could be determined.

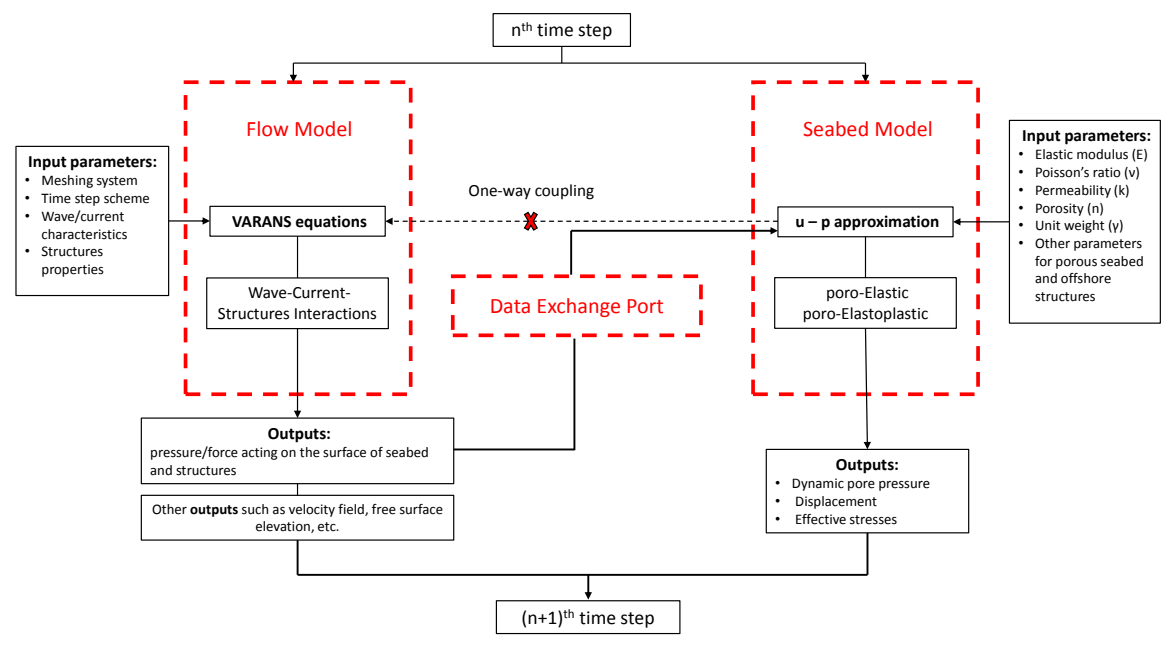

Fig. 1. The coupling process of integrated model. 


\section{Verification of the proposed model}

Before presenting parametric study, it is indispensable to validate the proposed model. In this section, the abilities of the proposed model to simulate the wavecurrent interactions and wave-submerged breakwater-seabed interactions are verified through two sets of published experimental data available in the literature.

\subsection{Comparison with Umeyama [2010]'s experiments of regular wave with uniform following current travelling over a rigid bottom}

In natural ocean environments, ocean waves and currents is commonly co-existed. In this section, the capability of flow model to simulate the interactions of wave and current over a rigid seabed foundation is verified by comparing the water surface elevation produced by numerical model and the previous experimental results [Umeyama, 2010].

Umeyama [2010] conducted a series of experiments to study the kinematic aspects of surface waves propagating with or without a steady current. A recirculating wave tank with length of $25.0 \mathrm{~m}$, width of $0.7 \mathrm{~m}$ and depth of $1.0 \mathrm{~m}$ was used in his experiments. The wave paddle was installed on the right hand side of the tank to generate waves. On the other side, a wave absorber was installed to avoid reflected waves. The water was recirculated via a pipe under the tank to generate a steady following current with the depth-average velocity of $U_{o}=8.0 \mathrm{~cm} / \mathrm{s}$. A probe was located at $14.0 \mathrm{~m}$ from the wave paddle to record the water surface elevation. The water depth was $30 \mathrm{~cm}$ and the wave period $(T)$ was $1.0 \mathrm{~s}$ during all the tests. Runs W1 to W3 were wave for loading only, while WC1 to WC3 were for combined wave-current loading. All the parameters used in these cases are list in Table 1.

Table 1. Parameters used in Umeyama [2010]'s experiments.

\begin{tabular}{lcccccc}
\hline & W1 & W2 & W3 & WC1 & WC2 & WC3 \\
\hline Depth average velocity $(\mathrm{cm} / \mathrm{s})$ & 0.0 & 0.0 & 0.0 & -8.0 & -8.0 & -8.0 \\
Wave height $(\mathrm{cm})$ & 1.03 & 2.34 & 3.61 & 0.91 & 2.02 & 3.09 \\
Wave period (s) & 1.0 & 1.0 & 1.0 & 1.0 & 1.0 & 1.0 \\
\hline
\end{tabular}

Figure 2 shows the comparison of phase-averaged water surface elevation between the numerical results and experimental results [Umeyama, 2010] in the wave alone cases and combined wave-current cases. Although the measured water surface elevation at the wave trough is slightly steeper than the simulation results for case 1 and case 3, overall, the agreement between numerical and experimental results is good. This demonstrates that the flow model is capable to simulate the interactions of wave and current. 

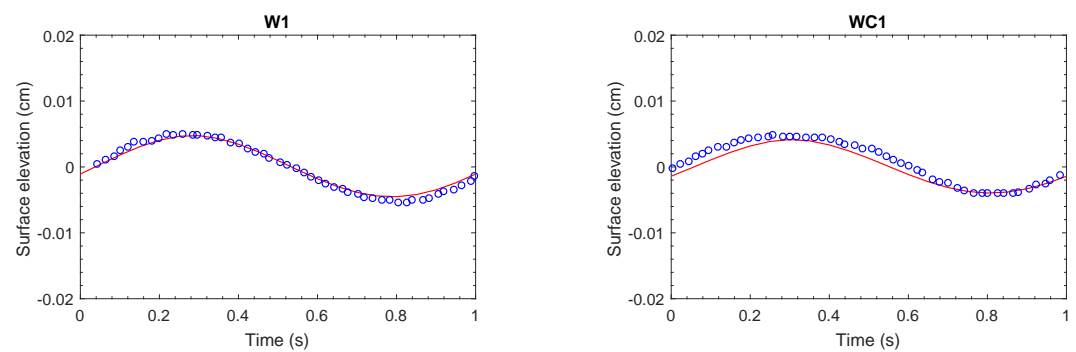

(a) case 1
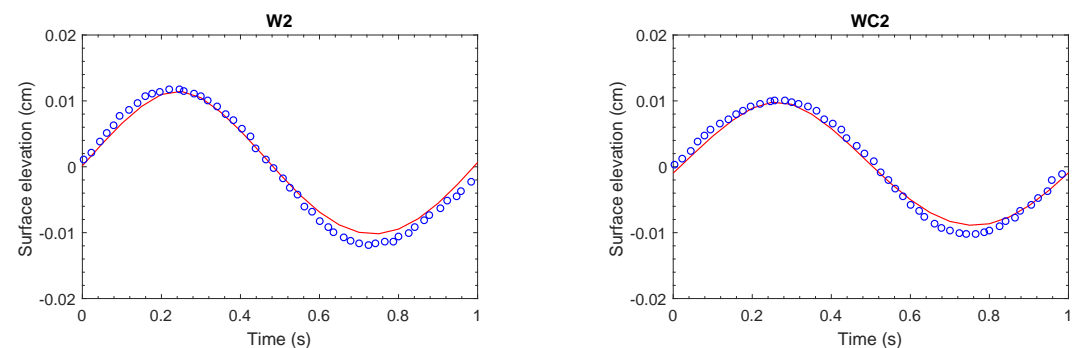

(b) case 2
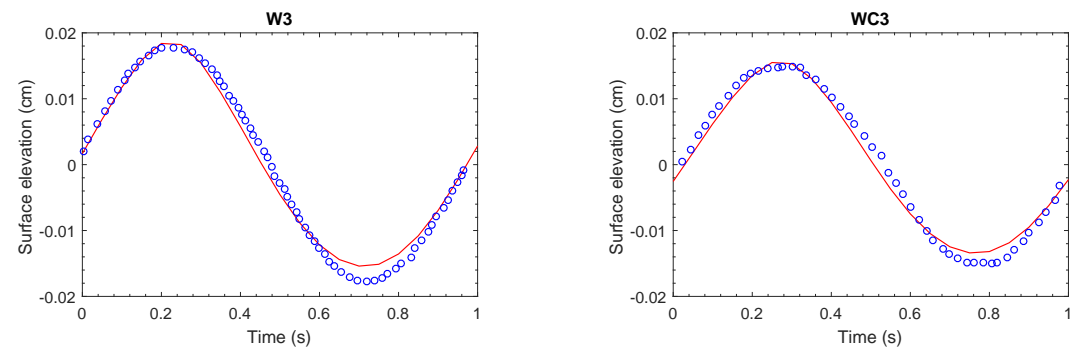

(c) case 3

Fig. 2. Comparison of water surface elevation between the simulation results and Umeyama [2010]'s experimental results. Solid line: simulation results; circle: experimental results.

\subsection{Comparison with Mizutani [1998]'s experiments of Submerged rubble mound breakwater}

In this section, the ability of the integrated model to simulate the wave-submerged porous breakwater-seabed interactions will be verified using Mizutani et al. [1998]'s experimental results. Mizutani et al. [1998] conducted a series of experiments to study the non-linear dynamic interaction between wave and permeable submerged breakwater over a finite thickness sand seabed. The set-up of these experiments are shown in Figure 3. The sandy seabed was $360.0 \mathrm{~cm}$ long and $19.0 \mathrm{~cm}$ thick. A trapezoid wooden step was placed on the left side of sandy seabed and a wave absorber was placed on the other side of seabed. The rubble mound breakwater with a $2: 1$ (horizontal:vertical) side slop was placed on the top middle of the seabed 
foundation. The crown of permeable breakwater was $105.0 \mathrm{~cm}$ in length and 21.0 $\mathrm{cm}$ in height. Four wave gauges were installed at Points a, b, c and d to monitor the wave height. Four pressure transducers were installed in the porous medium with one in breakwater (Point A) and three pore pressure transducers were installed in sandy seabed foundation (Points B, C and D) to record the dynamic pore pressures.

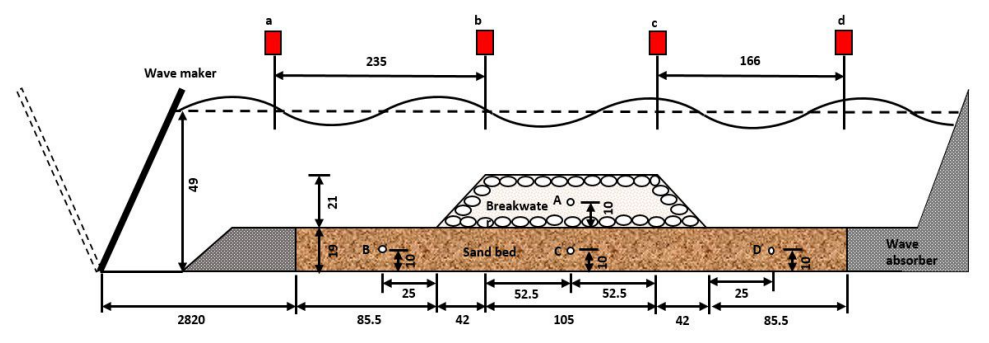

Fig. 3. The set-up of Mizutani [1998]'s experiments (units: cm).

The flow sub-model is validated by comparing the wave profiles at four monitory Points a, b, c and d and the seabed sub-model is verified by comparing the dynamic pressure at Points A, B, C and D with Mizutani et al. [1998]'s experimental results. Figure 4 shows the comparison of water profiles at Points a, b, c and d. For all four points (in front and behind the breakwater), the simulated results show a great consistency with Mizutani et al. [1998]'s experimental results. It can also be found that the submerged porous breakwater changes the pattern of wave propagation because that wave damping occurs over the breakwater crown. However, the wave sub-model can still accurately predict the water profiles. Figure 5 shows the comparison of the wave induced dynamic pressure at Points A, B, C and D. It is worth to notice that the sandy seabed and porous breakwater is treated as two different porous medium. The comparison demonstrates that the present model is capable to simulate the problems of wave-seabed-breakwater interactions. Detailed information of their experiments can be found in Mizutani et al. [1998].

\subsection{Comparison with Sassa and Sekiguchi [1999]'s geotechnical centrifuge test}

It is necessary to validate the capability of the present model to reproduced the residual soil response under cyclic waves loading, where the build-up of pore pressure takes place. Numerous studies regarding to wave-induced residual soil response were reported in the past few decades. Among them, Sumer et al. [2012] conducted a series of experiments in a wave flume with a soil pit to investigate the build-up of pore water pressure and resulting liquefaction of marine soils under progressive waves. The experimental data were also used to validate their mathematical model [Sumer and Fredsøe, 2002]. However, their experimental data are not used for the validation 


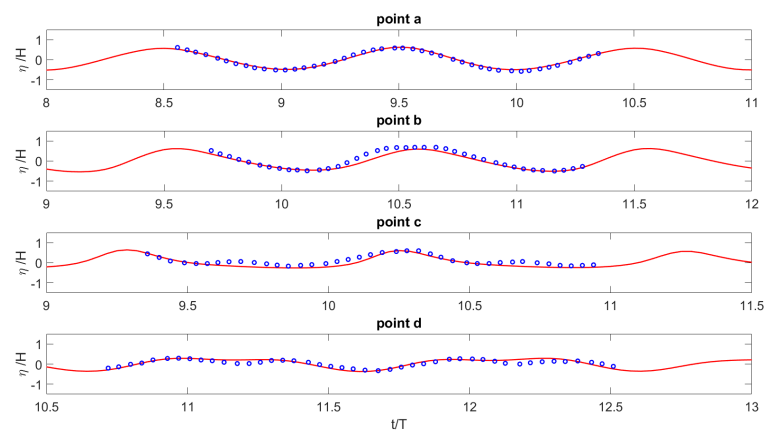

Fig. 4. Comparison between the simulation results and Mizutani [1998]'s experimental results for the water profile. Solid line: simulation results; circle: experimental results.

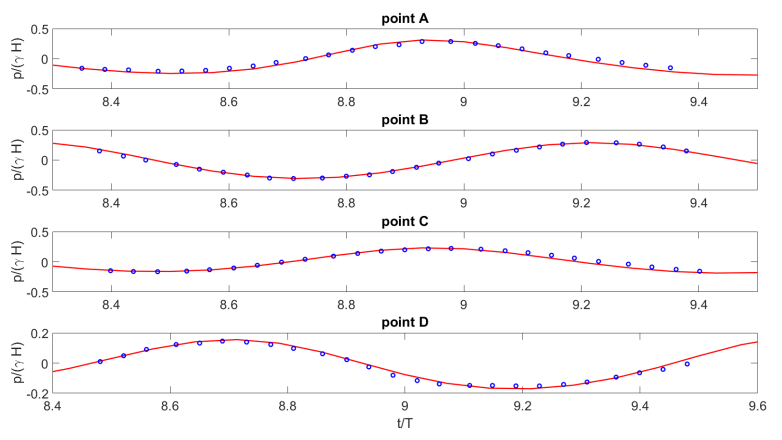

Fig. 5. Comparison between the simulation results and Mizutani [1998]'s experimental results for the wave induced dynamic pore pressure. Solid line: simulation results; circle: experimental results.

of the present model because some soil elasto-plastic parameters used in the present model are not available in their tests.

Another example is Sassa and Sekiguchi [1999]'s centrifuge tests. Three sets of centrifuge wave tank tests, including progressive- and standing-wave loading, with viscous scaling were performed by Sassa and Sekiguchi [1999] to investigate wave-induced liquefaction within loosely packed, fresh deposits of sandy bed. In this section, one of Sassa and Sekiguchi [1999]'s centrifuge tests (wave test P5-1) is used to verified the present model. The test was carried out under a steady-state centrifugal acceleration of $50 \mathrm{~g}$. The silicone oil with a viscosity of $50 \mathrm{cSt}$ was used as fluid. A sketch of Sassa and Sekiguchi [1999]'s test is shown in Figure 6.

Saturated Leighton Buzzard sand (British Standard sieve 100/700) was used in this test to form a soil bed with $200 \mathrm{~mm}$ width and $100 \mathrm{~mm}$ depth. It has following properties: specific gravity $G_{s}=2.65$, mean grain size $D_{50}=0.15 \mathrm{~mm}$, maximum void ration $e_{\max }=1.07$, minimum void ratio $e_{\min }=0.64$ and relative density $D_{r}$ 


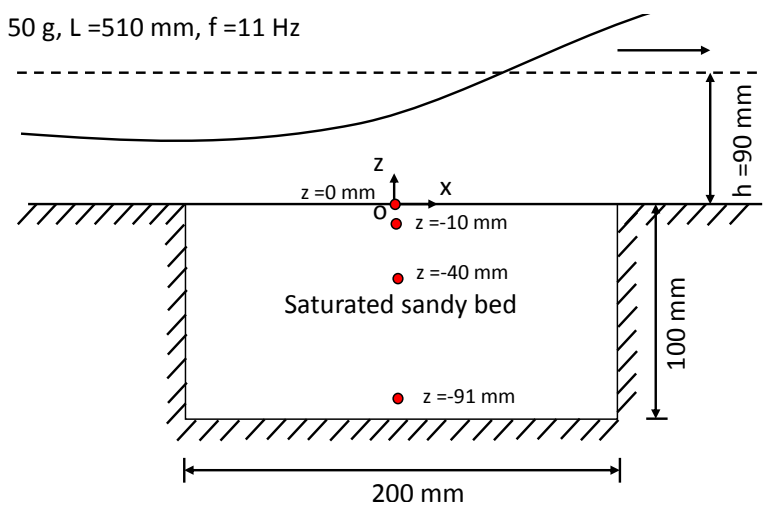

Fig. 6. Sketch of Sassa and Sekiguchi [1999]'s centrifuge test under progressive wave loading.

$=42 \%$. The waves were generated with following characteristic in centrifuge test: frequency $f=11 \mathrm{~Hz}$, water depth $d=90 \mathrm{~mm}$ and wave length $L=510 \mathrm{~mm}$, which will generate a $5.0 \mathrm{kPa}$ wave-induced pressure on seabed surface. This is equivalent to a wave with $4.5 \mathrm{~m}$ water depth, $4.55 \mathrm{~s}$ wave period and $1.7 \mathrm{~m}$ wave height in field for $1 \mathrm{~g}$ acceleration according to scaling principal [Sassa and Sekiguchi, 1999]. The parameters of the sand for PZIII model were identified by Sassa and Sekiguchi [2001]. The wave and soil parameters used in this verification are listed in Table 2.

In Sassa and Sekiguchi [1999]'s test, the wave-induced pore pressure changes in the soil bed were measured at four locations as indicated in Figure 6, placed in a vertical array in the centreline of the soil bed. Therefore, these four locations are selected as reference points to compare between results from the present model and Sassa and Sekiguchi [1999]'s data. Figure 7 shows that the numerical results from presented model agree well with Sassa and Sekiguchi [1999]'s centrifuge test results for wave-induced excess pore pressure in sandy seabed, which indicates that the present model is capable for simulating dynamic soil response within elasoplastic seabed foundation. It is worth to note that only the residual pore pressure of centrifuge test results is shown in Figure 7.

\section{Results and Discussions}

In this study, up to three permeable breakwaters with Bragg reflection are considered, as shown in Figure 8. This is the longitudinal section of breakwaters. The multiple breakwaters are sitting alongshore. Actually, there have been many research works regarding to multiple breakwaters [Zhang et al., 2012; Lan et al., 2011; Cho et al., 2004; Mase et al., 1996] in the literature. The effects of currents and the space between breakwaters on flow field properties and dynamic seabed response including soil liquefaction are investigated. As shown in Figure 8, $N(N=1,2$ and 3 ) denotes the number of submerged permeable breakwaters. Each breakwater has bottom width $W_{b}=24 \mathrm{~m}$, crown width $W_{t}=8 \mathrm{~m}$ and height $H=8 \mathrm{~m}$. The space 
Table 2. Parameters for verification with Sassa and Sekiguchi [1999]'s centrifuge test.

\begin{tabular}{lc}
\hline \multicolumn{2}{c}{ Wave characteristics } \\
\hline Wave height $\left(H_{w}\right)$ & $1.7 \mathrm{~m}$ \\
Wave period $(T)$ & $4.55 \mathrm{~s}$ \\
Water depth $(d)$ & $4.5 \mathrm{~m}$ \\
\multicolumn{2}{c}{ Soil characteristics } \\
\hline Poisson's ratio $\left(\mu_{s}\right)$ & 0.3 \\
Permeability $\left(k_{z}\right)$ & $1.5 \times 10^{-4} \mathrm{~m} / \mathrm{s}$ \\
Porosity $\left(n_{s}\right)$ & 0.445 \\
Degree of saturation $\left(S_{r}\right)$ & $100 \%$ \\
Relative density $\left(D_{r}\right)$ & $42 \%$ \\
\hline \multicolumn{2}{c}{ Parameters for PZIII model } \\
\hline$M_{g}$ & 1.2124 \\
$M_{f}$ & 0.75 \\
$\alpha_{f}$ & 0.1 \\
$\alpha_{g}$ & 0.1 \\
$K_{\text {evo }}$ & $660.8 \mathrm{KPa}$ \\
$G_{e s o}$ & $770.0 \mathrm{kPa}$ \\
$\beta_{0}$ & 0.2 \\
$\beta_{1}$ & 2.5 \\
$p_{0}^{\prime}$ & $4.0 \mathrm{kPa}$ \\
$H_{0}$ & 700.0 \\
$H_{U 0}$ & $1000.0 \mathrm{kPa}$ \\
$\gamma_{u}$ & 6.0 \\
$\gamma_{D M}$ & 4.0 \\
\hline \multicolumn{2}{c}{}
\end{tabular}

between two adjacent breakwaters is defined as $L$ and three sets of space ( $L=30$, 40 and $80 \mathrm{~m}$ ) are adopted. The rubble mound breakwaters are considered as elastic materials in this study, which plastic deformation is ignored within breakwaters. The properties of breakwaters are listed in Table 3 .

As mentioned before, the seabed foundation is treated as an elasto-plastic medium with the elasto-plastic model Paster-Zienkiewicz Mark-III Model adopted. The seabed foundation has a thickness of $h=20 \mathrm{~m}$ and length of $w=304 \mathrm{~m}$. The Nevada loose sand is used for seabed foundation with elasto-plastic soil behaviour and the property parameters are the same as those used in Zienkiewicz et al. [1999] which are determined through a series of dependent laboratory tests. For the detailed parameters, please refer to Table 3. As illustrated in Ye et al. [2013], for the soil model, the horizontal element size can be $L_{w} / 40$ and the vertical element size can be one-third to one-twice the size of horizontal. The time step is set as $\Delta t=T / N_{t}$, where $T$ is wave period and, in general, $N_{t}=40-50$. Therefore, the mesh sized selected for this soil foundation is $1 \mathrm{~m}$ in $z$-direction, $1 \mathrm{~m}$ and $2 \mathrm{~m}$ in $x$-direction for 
April 8, 2019 10:32 WSPC/INSTRUCTION FILE ijoce'lin'clean
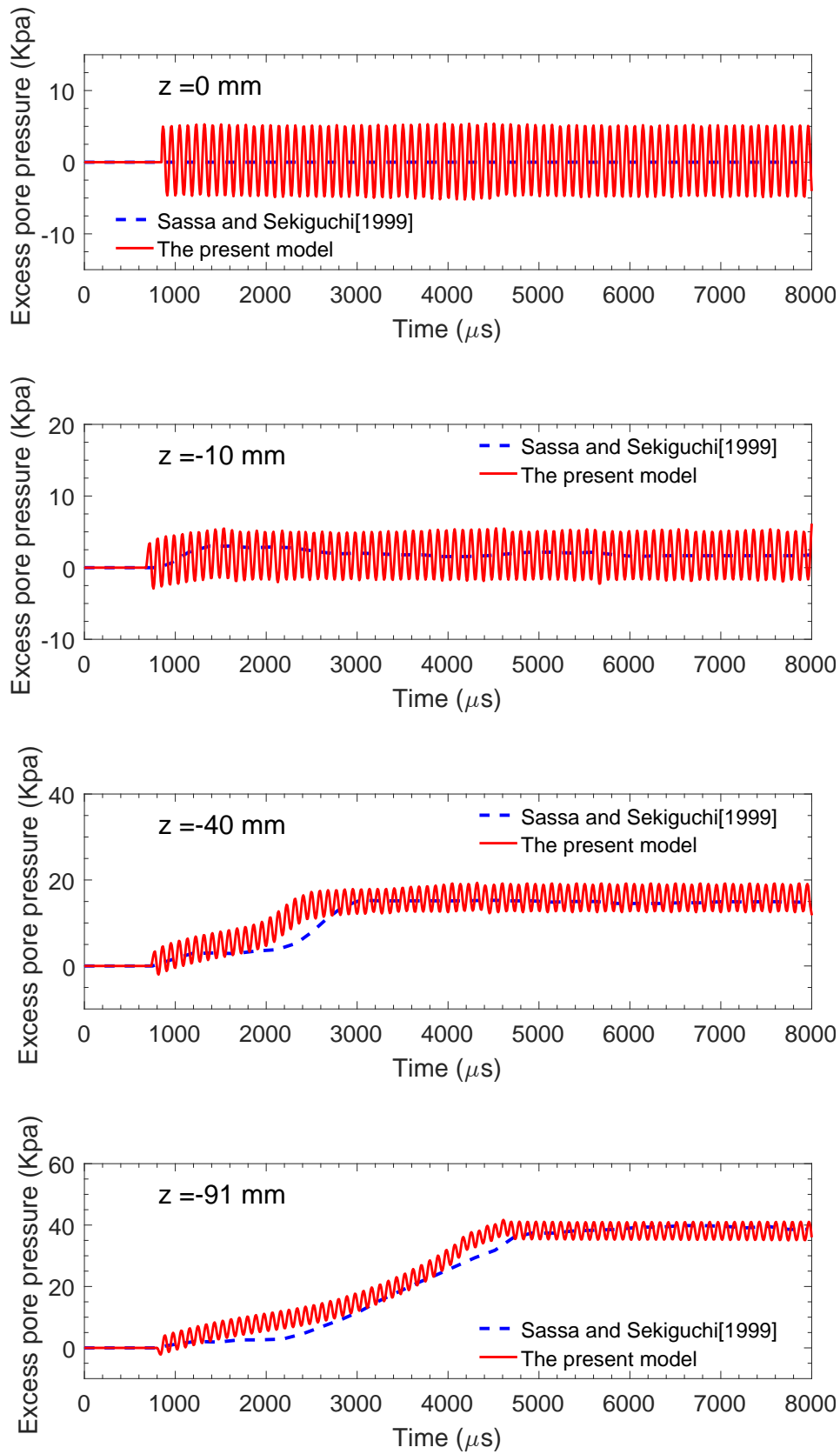

Fig. 7. Comparison between the simulation results and Sassa and Sekiguchi [1999]'s centrifuge test results for excess pore pressure in sandy seabed. Solid line: simulation results; dashed line: centrifuge test results.

the region around breakwaters and away from breakwaters, respectively. The time step is chosen as $0.2 \mathrm{sec}$. The mesh in the area around breakwaters are denser than 


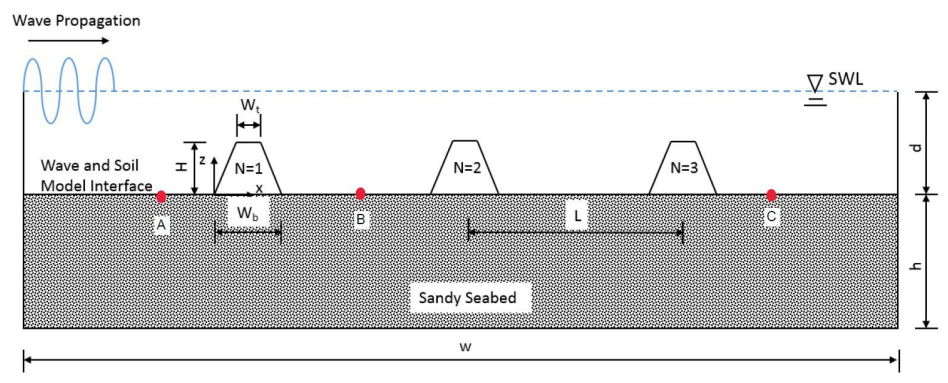

Fig. 8. Configuration of the numerical study.

the mesh in the area away from breakwaters. This allows the numerical model to better capture the flow-breakwater-seabed interactions.

Ocean waves with wave period $T=8 \mathrm{~s}$, wave height $H_{w}=3 \mathrm{~m}$ and water depth $d=15 \mathrm{~m}$ are generated at the left-hand side of the computational domain and propagating from left to right. Similar to the soil model, to obtain an accurate solution, the mesh size used in wave model varies from $L_{w} / 800$ to $L_{w} / 500$ in the $x$-direction with a refinement factor of 2 , which is the ratio between the mesh size of the area without breakwater and the area in the vicinity of the structure. The vertical mesh size is adopted as one-fifth of the horizontal mesh size.

\subsection{Flow field involved in wave-current submerged breakwaters interactions}

Submerged permeable breakwaters have been widely used in ocean engineering projects to protect coast lines by dissipating or breaking the waves that pass over it. Most previous studies focused on hydrodynamic properties around a single breakwater under only wave loading without currents [Mizutani et al., 1998; Mostafa et al., 1999]. The construction of a breakwater will change the flow pattern in vicinity by wave shoaling, reflection and diffraction, which makes the hydrodynamic properties around breakwater to be complicated. This becomes even worse when multiple breakwaters are presented which will amplify the reflected waves, known as Bragg reflection. In this section, the effects of number of breakwaters, space between breakwaters and presence of current on hydrodynamic properties around breakwaters will be examined using the flow model.

Figure 9 compares the time series of water surface elevation at three typical locations $(x=-8 \mathrm{~m}, 32 \mathrm{~m}, 112 \mathrm{~m})$ among cases with different number of breakwaters $(N=1,2$ and 3$)$. For the multiple breakwater cases, the spacing between the breakwaters is $L=40 \mathrm{~m}$. The first and second locations are in front of the first and second breakwaters respectively and the third location is behind the third breakwater. Cases with one and two breakwaters are realised by removing second and/or third breakwaters from case with three breakwaters. As shown in the figure, the 
Table 3. Input data for the parametric study.

\begin{tabular}{|c|c|}
\hline \multicolumn{2}{|l|}{ Wave characteristics } \\
\hline Wave height $\left(H_{w}\right)$ & $3.0 \mathrm{~m}$ or various \\
\hline Wave period $(T)$ & $8.0 \mathrm{~s}$ or various \\
\hline Still water level $(d)$ & $15.0 \mathrm{~m}$ or various \\
\hline \multicolumn{2}{|l|}{ Soil characteristics } \\
\hline Poisson's ratio $\left(\mu_{s}\right)$ & 0.333 \\
\hline Permeability $\left(k_{z}\right)$ & $1.0 \times 10^{-5} \mathrm{~m} / \mathrm{s}$ or various \\
\hline Porosity $\left(n_{s}\right)$ & 0.3 \\
\hline Degree of saturation $\left(S_{r}\right)$ & $98 \%$ or various \\
\hline Relative density $\left(D_{r}\right)$ & $40 \%$ \\
\hline \multicolumn{2}{|c|}{ Breakwater characteristics } \\
\hline Young's modulus $\left(E_{s}\right)$ & $1.0 \times 10^{6} \mathrm{~Pa}$ \\
\hline Poisson's ratio $\left(\mu_{s}\right)$ & 0.33 \\
\hline Permeability $\left(k_{z}\right)$ & $8.9 \times 10^{-2} \mathrm{~m} / \mathrm{s}$ \\
\hline Porosity $\left(n_{s}\right)$ & 0.5 \\
\hline Degree of saturation $\left(S_{r}\right)$ & $99.98 \%$ \\
\hline \multicolumn{2}{|c|}{ Parameters for Nevada loose sand } \\
\hline$M_{g}$ & 1.15 \\
\hline$M_{f}$ & 1.035 \\
\hline$\alpha_{f}$ & 0.45 \\
\hline$\alpha_{g}$ & 0.45 \\
\hline $\bar{K}_{\text {evo }}$ & $770.0 \mathrm{kpa}$ \\
\hline$G_{\text {eso }}$ & $1155.0 \mathrm{kpa}$ \\
\hline$\beta_{0}$ & 4.2 \\
\hline$\beta_{1}$ & 0.2 \\
\hline$p_{0}^{\prime}$ & $4.0 \mathrm{kpa}$ \\
\hline$H_{0}$ & 600.0 \\
\hline$H_{U 0}$ & $4000.0 \mathrm{kpa}$ \\
\hline$\gamma_{u}$ & 2.0 \\
\hline$\gamma_{D M}$ & 0.0 \\
\hline
\end{tabular}

water profile oscillates over time at three locations. As the location goes from left to right, the amplitudes decrease in sequence due to the blockage effect of breakwaters. For the first location $(x=-8 \mathrm{~m})$, which is in front of the first breakwater, the water profiles for three cases are almost identical. While the water profile at second location $(x=32 \mathrm{~m})$ is obviously smaller in the case with one breakwater than the one with two/three breakwaters. This is because that for the latter two 
cases, the wave reflects back by the second/third breakwater and makes water profile become higher. For the location behind the third breakwater, the wave height becomes smaller successively in the cases with one, two and three breakwaters. This demonstrates that the multiple submerged breakwaters can dissipate the propagating waves more efficiently and better protect the coastline.

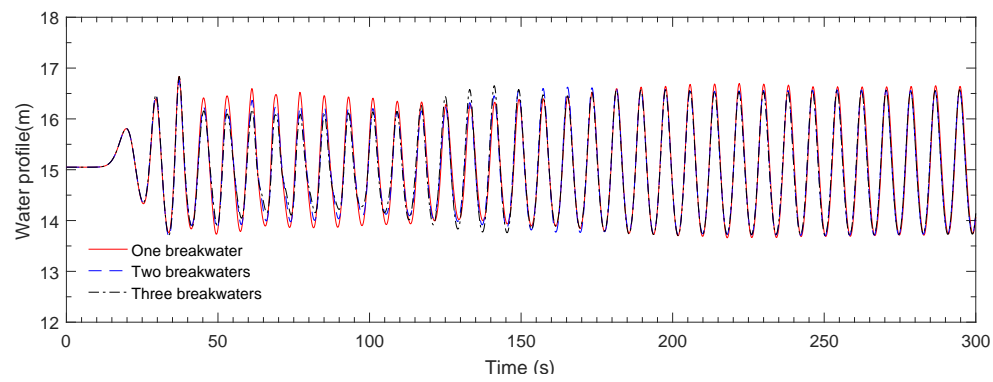

(a) $x=-8 \mathrm{~m}$

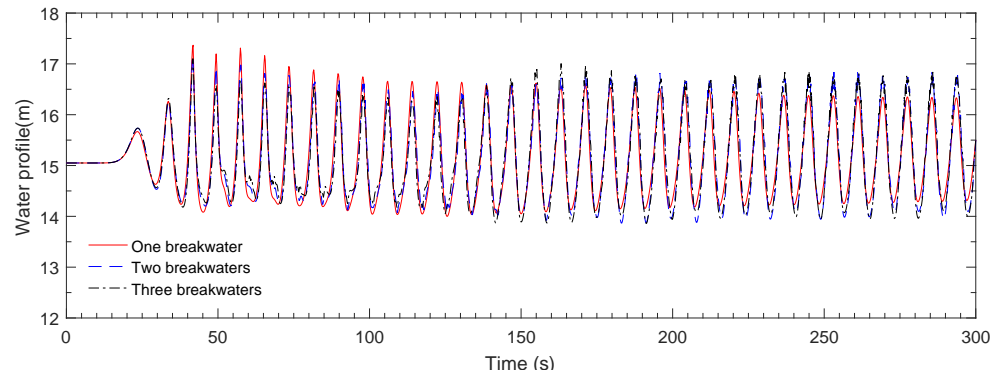

(b) $x=32 \mathrm{~m}$

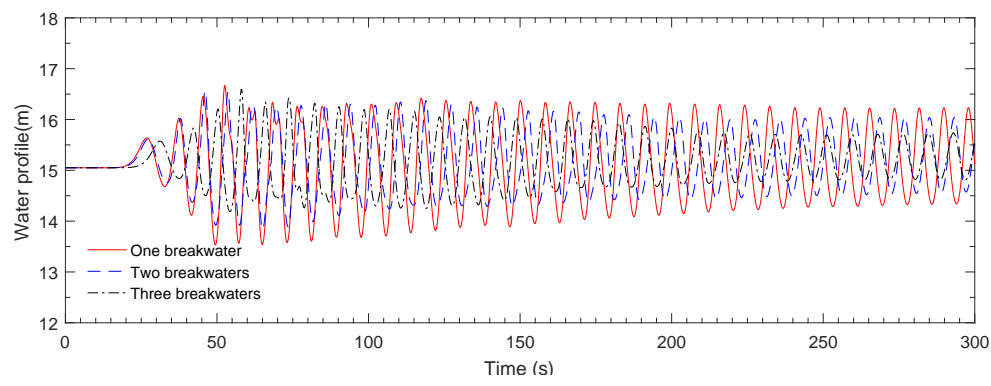

(c) $x=112 \mathrm{~m}$

Fig. 9. Time series of water profiles at three typical locations for the cases with one, two and three breakwaters.

Figure 10 shows the time series of water profiles at two typical location (in front of first breakwater and behind the last breakwater) under three conditions (the presence of following current $U_{0}=1 \mathrm{~m} / \mathrm{s}$, without current $U_{0}=0 \mathrm{~m} / \mathrm{s}$ and the presence of opposing current $U_{0}=-1 \mathrm{~m} / \mathrm{s}$ ). Similar, the water profile attenuates from $x=-8 \mathrm{~m}$ to $x=112 \mathrm{~m}$ due to the blockage of breakwater group. As can be easily seen 
from the figures, the presence of the current significantly affects the wave shapes. The following current causes phase leading while the opposing current causes phase lag. At the location in front of first breakwater $(x=-8 \mathrm{~m})$ with less impact of breakwater, the wave height tends to be amplified in the opposing current case in comparison with the cases with following current or without current. However, at the second location $(x=112 \mathrm{~m})$ which is behind the last breakwater, due to the reason that the following current prohibits the wave energy dissipation by breakwaters while the opposing current enhances this ability, the wave height is larger in the leeward side of breakwaters in the following current case. Therefore, the presence of current interacting with submerged breakwaters can significantly change the shape of the propagation waves and further affect the seabed stability.

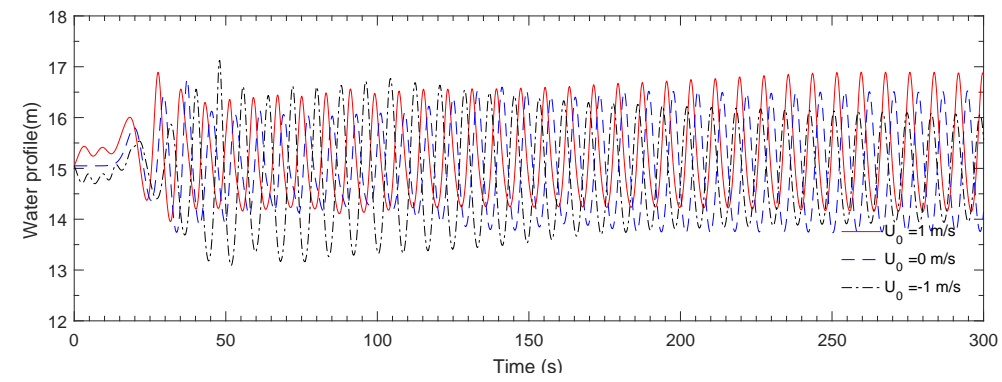

(a) $x=-8 \mathrm{~m}$

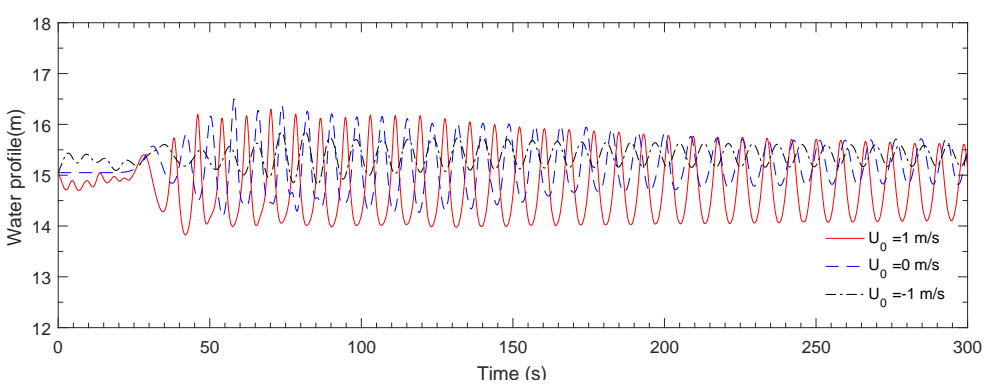

(b) $x=112 \mathrm{~m}$

Fig. 10. Time series of wave profiles at two typical locations for the cases under following current, no current and opposing current.

Distributions of horizontal velocity field at $t=240 \mathrm{~s}$ for the cases with different distance between adjacent breakwaters $(L)$ are shown in Figure 11. The values of $L$ are equal to $40 \mathrm{~m}, 80 \mathrm{~m}$ and $30 \mathrm{~m}$ respectively. Using linear wave theory, the wavelength is calculated as $81.75 \mathrm{~m}$. The horizontal velocity field shows a positive and negative alternation and the horizontal velocity decreases with an increasing of water depth in $z$-direction. In the region in front of breakwaters, the horizontal velocity appears as negative due to the wave reflection. Owning to the blockage effect of breakwaters, the magnitude of the horizontal velocity decreases significantly at 
the shoreward side of last breakwater. Comparing three cases with different value of $L$, the magnitudes of the horizontal velocity in the case with $L=40 \mathrm{~m}$ are larger at the seaward side but smaller at the shoreward side than the ones in case with $L=80 \mathrm{~m}$ and $L=30 \mathrm{~m}$. This is attribute to that a stronger Bragg reflection takes place in the first case, which the wave length is twice as long as the distance between two adjacent breakwaters. Mei et al. [2005] pointed out that the phenomenon Bragg reflection becomes stronger when the wave length of surface wave becomes closer to the twice of distance of bottom undulation. The stronger Bragg effect will be benefit to the protection of shoreline. On the other hand, stronger Bragg effect will imply more significantly chance for the seabed foundation to be liquefied as the stronger reflection makes the wave height larger in front of breakwaters. As the result, this study will focus on the condition with $L=40 \mathrm{~m}$ that causes the strongest Bragg effect.

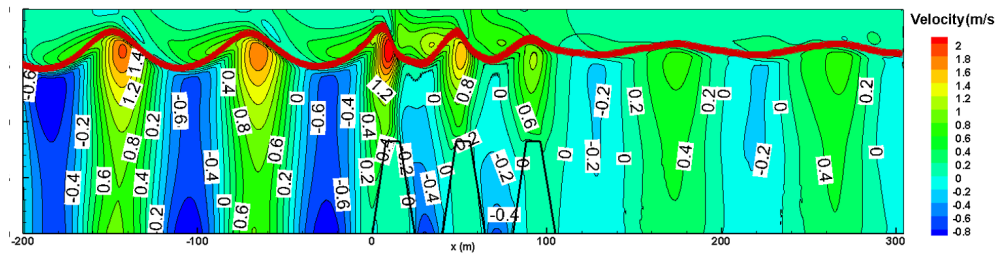

(a) $L=40 \mathrm{~m}$

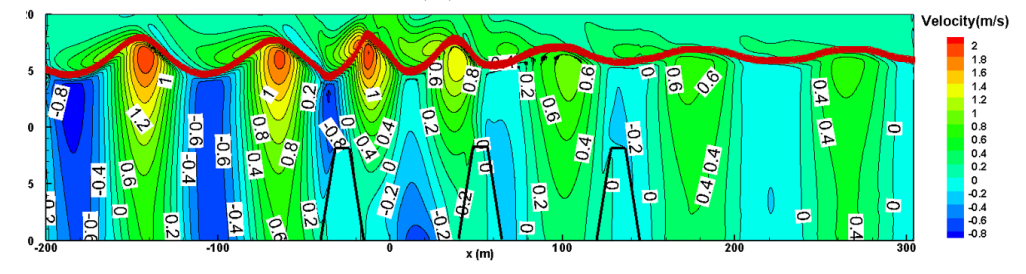

(b) $L=80 \mathrm{~m}$

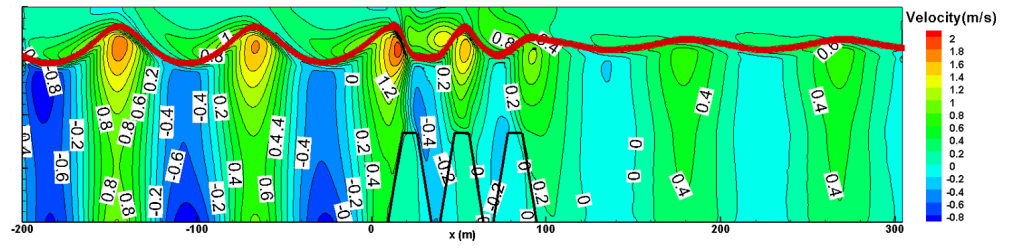

(c) $L=30 \mathrm{~m}$

Fig. 11. Distributions of the horizontal velocity field at $t=240 \mathrm{~s}$ for the cases with different distance between adjacent breakwaters.

\subsection{Consolidation of seabed foundation}

In natural environments, a seabed foundation is consolidated under hydro-static pressures from the seawater above and self-weight in the geological history. Con- 
struction of breakwaters will change the stress field in seabed foundation around breakwaters. The excess pore water pressure can be generated. A new balance condition will be reached with the dissipation of excess pore pressure within the seabed foundation. To better simulate the wave-breakwaters-seabed interactions, this new balance state will be determined first and used as the initial conditions for the later dynamic soil analysis. The consolidation process is completed when the distribution of pore pressure becomes uniform and layered. At this stage, the seabed foundation becomes stable again under static seawater loading and self-gravity from breakwaters. The distributions of pore pressure, effective normal stress and shear stress within the seabed foundation and permeable breakwaters after completion of consolidation process are shown in Figure 12.
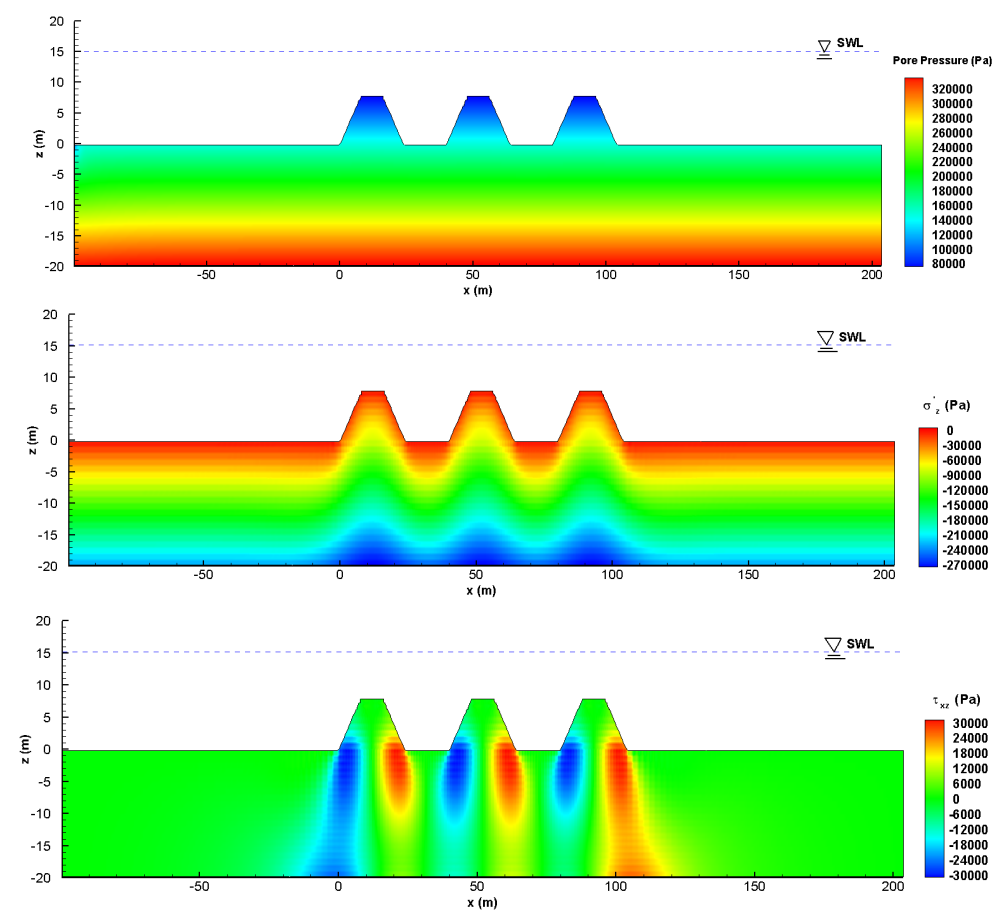

Fig. 12. Distributions of pore pressure, effective normal stress and shear stress in the seabed foundation after consolidation is completed.

As shown in Figures 12, the construction of breakwaters has significantly affected the effective normal stress $\left(\sigma_{z}^{\prime}\right)$ and shear stress $\left(\tau_{x z}\right)$ in the region near breakwaters. The magnitude of effective normal stress $\left(\sigma_{z}^{\prime}\right)$ obviously increases under each breakwater and appears as layered structure, which is caused by the gravity of each breakwater. The concentration zones of shear stress appear in the area under two sides of each breakwater. Additionally, it can be seen from Figure 12 that shear concentration zones under the second breakwater and inside of first/last breakwaters 
are much smaller than the shear concentration zones under the outside of first/last breakwaters. This is because soil in the area is denser than the soil in later mentioned area due to the self-weight of breakwaters. Therefore, the shear failure is less likely to happen in this area. This phenomenon depends on the space between two adjacent breakwaters. It is also worth to point out that the stress field in the region far away from breakwaters have not been affected. The pore pressures in the computational domain increase uniformly from top to bottom, indicating that no excess pore pressure exists. This final state after consolidation process will be used as initial state for dynamic analysis of seabed model.

\subsection{Dynamic response of loosely deposited seabed foundation}

In this section, the behaviour of loosely deposited sandy seabed foundation around submerged breakwaters group under combined wave and current loading will be discussed.

\subsubsection{Effective normal stress, shear stress and pore pressure}

Effective normal stresses, shear stresses and pore pressures are important parameters to understand dynamic response of loosely deposited seabed foundation under combined wave and current loading. Figure 13 illustrates the time series of wavecurrent induced effective normal stress, shear stress and pore pressure at two typical locations: A $(x=-1 \mathrm{~m}, z=-4 \mathrm{~m})$ and $\mathrm{B}(x=105 \mathrm{~m}, z=-4 \mathrm{~m})$ which are in front of first breakwater and behind the last breakwater, respectively. It is observed from Figure 13 that the dynamic responses of seabed foundation consist of two components: oscillatory component and residual component. The oscillatory part fluctuates with propagating wave, whose temporal average over wave cycle equals to zero. The residual part is caused by the volumetric contraction of sandy soil under cyclic wave loading.

The pore pressures at both locations build up progressively with time. However, it is not unlimited, it remains a constant after many wave loading cycle at location A (in front of first breakwater). This constant is the critical value $P_{c r}$ of soil liquefaction. Sumer $[2013 ; 2014]$ pointed out the criterion of onset of liquefaction: when the built-up pore-water pressure reaches a critical value, the soil will liquefy. Herein, $P_{c r}$ is the initial mean normal effective stress after the pre-consolidation process has been completed:

$$
\sigma_{0}^{\prime}=\frac{1+\mu_{s}}{3}\left(\sigma_{x 0}^{\prime}+\sigma_{z 0}^{\prime}\right)
$$

in which $\mu_{s}$ is the Poisson's ratio; $\sigma_{x 0}^{\prime}$ and $\sigma_{z 0}^{\prime}$ are the horizontal and vertical initial effective stresses in elasto-plastic seabed foundation determined through consolidation process under the gravitational force of multiple breakwaters, respectively. 
In the case of seabed foundation without a marine structure, the initial mean normal effective stress can be defined as:

$$
\sigma_{0}^{\prime}=\frac{1+2 k_{0}}{3} \gamma^{\prime} z
$$

in which $z$ is the depth of the measured point from surface of seabed foundation downwards; $\gamma^{\prime}$ is the submerged specific weight of soil, defined as: $\gamma^{\prime}=\gamma_{\text {soil }}-\gamma_{\text {water }}$; $k_{0}$ is the coefficient of lateral earth pressure at rest.

Along with the build up of pore pressures, the effective normal stresses and shear stresses decrease, and approaching to zero. This is because that when the pore pressure builds up, the contacted effective stresses between the soil particles become smaller, the soil tends to a status of liquid. As can be seen at location A, when the built-up pore pressures reached the critical value $P_{c r}$, the soil becomes liquefied. At the same time, the effective normal stresses and shear stress both become zero. The reason of this phenomenon is that when liquefaction occurs, the soil behaves as liquid, the contacted effective stress become zero and can not bear any shear stresses.

Comparing the results at locations $\mathrm{A}$ and $\mathrm{B}$, it can be seen that the effective normal stresses and shear stresses reached zero, built-up pore pressure reached critical value $P_{c r}$ after many wave cycle, which means that soil at location A has liquefied; while at location $\mathrm{B}$, the effective normal stresses and shear stresses still remain a relative large value, the built-up pore pressure did not reach critical value $P_{c r}$, which indicates that the soil at location $\mathrm{B}$ has not liquefied. The reason is that location $\mathrm{A}$ is in front of first breakwater and location B is behind last breakwater. Due to the hydrodynamic function of multiple breakwaters (mainly because of shoaling and Bragg reflection), the hydrodynamic pressure at location $\mathrm{A}$ is much larger than the one at location B, which makes location A much easier to be liquefied. For the locations beneath the multiple breakwaters, the gravity of the breakwaters compress the seabed foundation, makes the effective stresses at these locations much more difficult to become zero, in other words, more difficult to be liquefied. Another interesting aspect is that the oscillatory part of the pore pressure is relative small at location B. This also attribute to the protection of breakwaters.

Another factor that influence the dynamic soil response is the presence of currents. As shown in Figure 13, three current conditions are considered: following current $\left(U_{0}=1 \mathrm{~m} / \mathrm{s}\right)$, without a current $\left(U_{0}=0 \mathrm{~m} / \mathrm{s}\right)$ and opposing current $\left(U_{0}=-1\right.$ $\mathrm{m} / \mathrm{s})$. It can be clearly seen from the figures that presence of current significantly affects the dynamic soil response in the elasto-plastic seabed foundation: compared to the case without current $\left(U_{0}=0 \mathrm{~m} / \mathrm{s}\right)$, the following current $\left(U_{0}=1 \mathrm{~m} / \mathrm{s}\right)$ aggravates the soil response and brings forward the occurrence of soil liquefaction while the opposing current $\left(U_{0}=-1 \mathrm{~m} / \mathrm{s}\right)$ mitigates the soil response and postpone the occurrence of soil liquefaction. For example, for location A, the soil liquefied at around 240 s, 320s and 440s for the cases with following current, without a current and with an opposing current respectively. The existence of a current will change the wave 
characteristics. The following current could significantly elongate the wave length, decrease wave number and increase the maximum pressure that acting on seabed. While the opposing current has the opposite effect and make the maximum pressure acting on seabed decrease. As one-way coupling algorithm is adopted between fluid and seabed model. Only the hydrodynamic pressure from fluid model will be applied on seabed surface. Therefore, the effects of a current on soil response are reflected in effect of hydrodynamic pressure acting on seabed surface. The hydrodynamic pressure acting on seabed surface increases in following current cases, resulting in higher potential of seabed instability. As the result, a conclusion can be made that the opposing current, to some extents, can avoid occurrence of soil liquefaction while following current has negative effects on seabed foundation stability. In addition, it can be seen from the figures that the following current magnifies the oscillatory part of soil response while the opposing current has opposite effects.
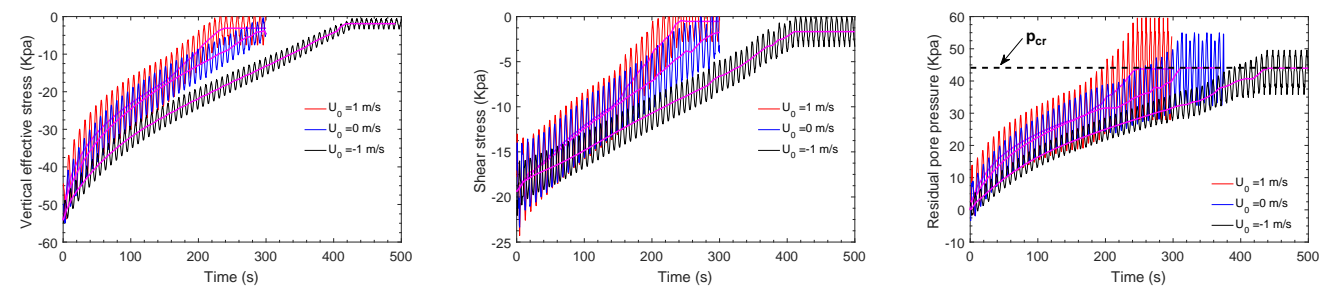

(a) Location $\mathrm{A}(x=-1 \mathrm{~m}, z=-4 \mathrm{~m})$ (in front of first breakwater)
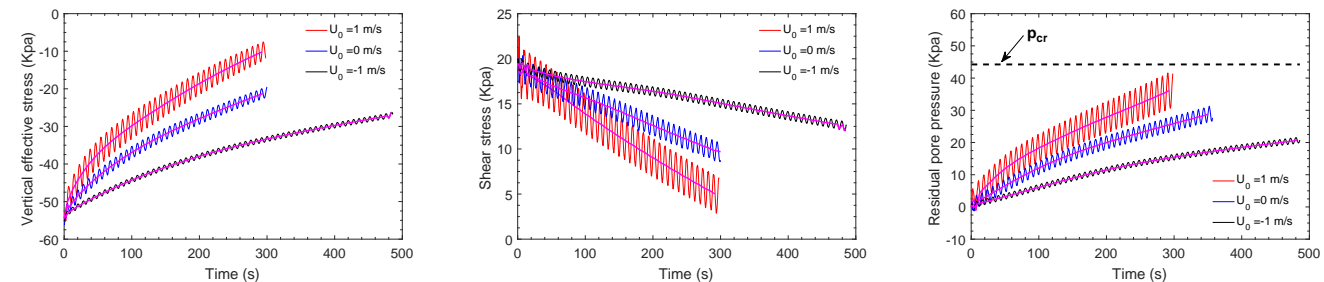

(b) Location B $(x=105 \mathrm{~m}, z=-4 \mathrm{~m})$ (behind last breakwater)

Fig. 13. Time series of wave-current induced reduction of effective normal stress and shear stress and build up of pore pressure at two typical locations in a sandy seabed foundation.

\subsubsection{Displacements}

Another important indicator to understand dynamic soil response in an elastoplastic seabed foundation around multiple breakwaters under combined wave and current loading is soil displacement. Note that the liquefied soil behaves like heavy fluid. Under combined wave-current loading and self-weight of multiple breakwaters, this kind of heavy fluid vibrates along with the wave-current motion, which generates permanent deformations within the elasto-plastic seabed foundation. Figure 14 
illustrates the time series of wave-current induced displacement in an elasto-plastic seabed foundation at $z=-4 \mathrm{~m}$. Three typical locations are selected: $x=-1 \mathrm{~m}, x=52$ $\mathrm{m}$ and $x=105 \mathrm{~m}$, which are in front of first breakwater, right beneath the second breakwater and behind the last breakwater respectively. It can be observed from the figure that there is obvious lateral spreading in the loosely deposited sandy seabed at location $x=-1 \mathrm{~m}$ and $x=105 \mathrm{~m}$. Compare the horizontal displacement at three locations, it can be found that at $t=300 \mathrm{~s}$, lateral spreading reaches $-13 \mathrm{~cm}$ at $x=-1$ $\mathrm{m}$ and $6 \mathrm{~cm}$ at $x=105 \mathrm{~m}$. While at $x=52 \mathrm{~m}$, there is no lateral spreading happens, it vibrates around 0 . This is because that the soil at location $x=52 \mathrm{~m}$ is relatively compacted soil due to gravity of the breakwater. The deformation and displacement are more difficult to happen in this denser soil. Furthermore, the multiple breakwaters have a certain extent protection for soil deformation behind breakwaters, compared between $x=-1 \mathrm{~m}$ and $x=105 \mathrm{~m}$.

The vertical displacement shows a different trend: there are significant vertical subsidence at all three locations with around $4.8 \mathrm{~cm}, 1.8 \mathrm{~cm}$ and $1.5 \mathrm{~cm}$ at $x=52 \mathrm{~m}$, $x=-1 \mathrm{~m}$ and $x=105 \mathrm{~m}$ respectively. Similarly, due to the self-weight of breakwater, location $x=52 \mathrm{~m}$ has most obvious sink. A very interesting phenomenon can be observed at $x=-1 \mathrm{~m}$ : the soil keeps subsiding until around $t=220 \mathrm{~s}$, then an upward displacement appears. This is mainly because that location $x=-1 \mathrm{~m}$ is just in front of first breakwater, the soil under first breakwater become liquefied at $t=220 \mathrm{~s}$, after liquefaction happens, the soil under first breakwater can not support the first breakwater above, the first breakwater suddenly has a large subsidence, this kind of squeezing results upward movement of surrounding soil. It may be concluded that phenomenon showing an upward movement after subsidence of soil could be used as an indirect indicator of soil liquefaction happening under the breakwater. Furthermore, the oscillatory of displacement is larger in front of breakwaters compared to the locations behind breakwaters.
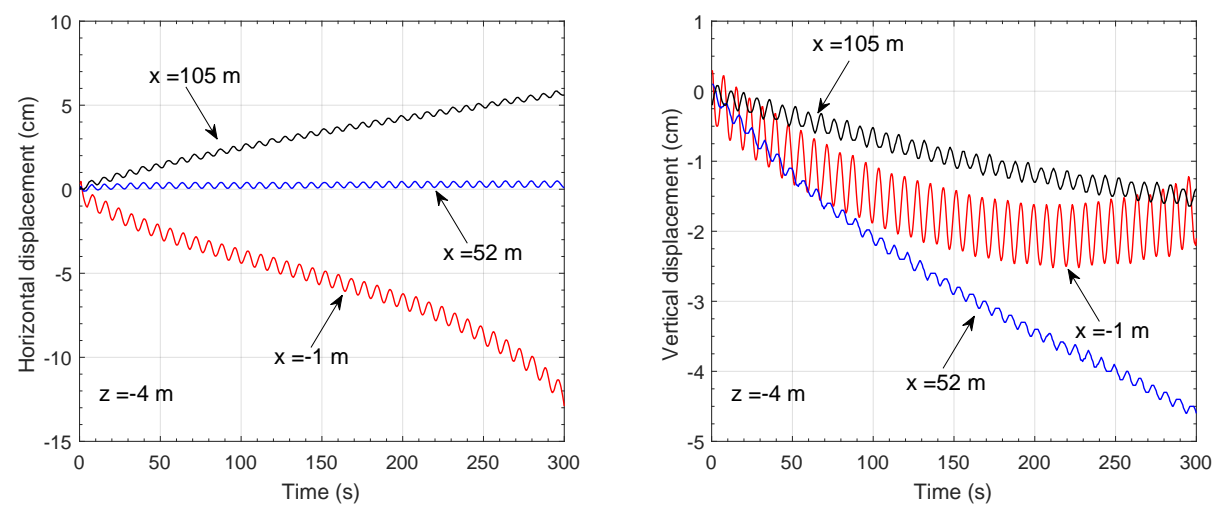

Fig. 14. Time series of wave-current induced displacement in seabed foundation at $z=-4 \mathrm{~m}$. 


\subsubsection{Residual liquefaction}

In general, two types of soil liquefaction mechanisms exist: momentary liquefaction and residual liquefaction Sumer [2014]. The former only occurs in very dense soil during the passage of the wave trough, which has minor effects on the stability of coastal structures. However, momentary liquefaction could deteriorate the scouring around offshore structures. Residual liquefaction has critical effects on coastal structures stability. As demonstrated previously, the seabed foundation soil is softening during the build up process of pore pressure. When pore pressure reaches critical value $P_{c r}$, the effective stresses between the soil particles decrease to zero, the residual liquefaction occurs. Soil behaves like liquid and looses its bearing capacity. Under this circumstance, the offshore structures are very likely to collapse. Therefore, evaluation of the liquefaction potential of seabed foundation is essential for structures design and maintaining. Because the PZIII model cannot describe the mechanical behaviour of sandy soil under tensile stress and zero stress, the PZIII model is not suitable for liquefied sandy soil with zero contact stress. In this study, only the liquefaction potential is included without considering the post-liquefaction behaviour. In other words, this study focus on the soil behaviour up to the onset of liquefaction. To predict the process of post-liquefaction, the model proposed by Sassa et al. [2001] and Liu et al. [2009] should be used. However, their models were based on one-dimensional approach, which is only valid for the wave-seabed interactions without a structure. To the authors' best knowledge, there is no reference available in the literature for the post-liquefaction with a structure (i.e., 2D or 3D cases). Further development of the numerical model is required to describe post-liquefaction soil behaviour. The sequence of soil behaviour during wave-induced liquefaction was presented by Sumer et al. [2006]. For the full description of these processes, readers can refer to Sumer et al. [2006]. In this section, the wave-current induced residual liquefaction potential in an elasto-plastic seabed foundation with three breakwaters built on it will be quantitatively investigated.

Based on the liquefaction criterion proposed by Okusa [1985], a parameter named liquefaction potential is defined as below [Ye, 2012]:

$$
L_{\text {potential }}=\frac{\sigma_{z d}^{\prime}}{\left|\sigma_{0}^{\prime}\right|}
$$

where $\sigma_{z d}^{\prime}$ and $\sigma_{0}^{\prime}$ are the wave-current induced effective normal stress and initial mean normal effective stress, respectively.

As the sandy soil can not bear any tensile stress as silty or cohesive soil, the liquefaction potential for sandy soil can not exceed 1.0. Jiaer et al. [2004] suggested an adjustment coefficient $\alpha_{r}$, range from 0.78 to 0.99 depending on the soil characteristics, for the occurrence of liquefaction in sandy soil. In this study, we assume the sandy soil liquefied when $L_{\text {potential }}$ reaches 0.9 [Ye, 2012].

Figure 15 shows the time series of liquefaction potential at four location $(x=-1$ $\mathrm{m}, x=32 \mathrm{~m}, x=52 \mathrm{~m}$ and $x=105 \mathrm{~m})$ at two depths $(z=-4 \mathrm{~m}$ and $z=-6 \mathrm{~m})$, 
respectively. It can be seen from the figure that liquefaction potential increases over time at all locations. Horizontally, the order of four location that with largest liquefaction potential to smallest liquefaction potential is: $x=-1 \mathrm{~m}, x=105 \mathrm{~m}, x$ $=32 \mathrm{~m}$ and $x=52 \mathrm{~m}$. The reason, as mentioned above, is that location $x=52 \mathrm{~m}$ is beneath the second breakwater, location $x=-1 \mathrm{~m}$ is in front of the first breakwater, the compression of the breakwater makes the growth rate of liquefaction potential at locations beneath and behind the breakwater much less than that in front of breakwaters. Vertically, with the increase of soil depth, the liquefaction potential decreases because the initial stress increased as soil depth increases. This indicates that the liquefaction is more likely to happen in the upper layer of seabed foundation.

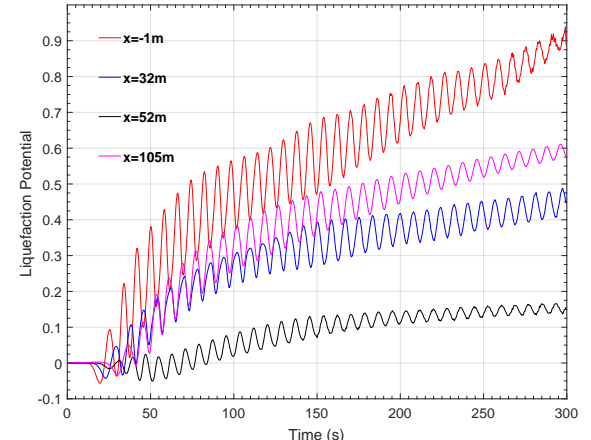

(a) $z=-4 \mathrm{~m}$

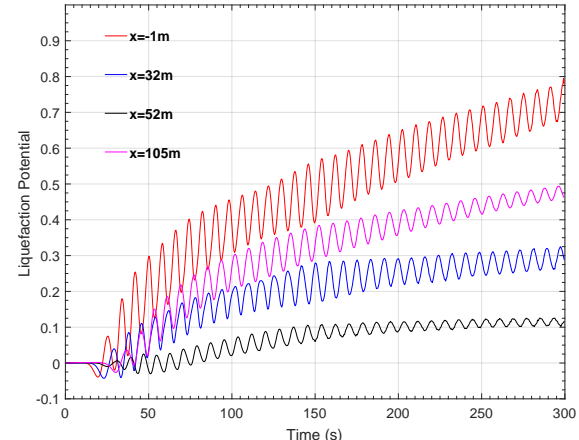

(b) $z=-6 \mathrm{~m}$

Fig. 15. Time series of liquefaction potential at typical locations around breakwaters, (a) $z=-4 \mathrm{~m}$; (b) $z=-6 \mathrm{~m}$.

Figure 16, 17 and 18 show the wave-current induced maximum liquefaction areas around one, two and three breakwaters respectively at three typical times $(t=100 \mathrm{~s}$, $t=200 \mathrm{~s}$ and $t=300 \mathrm{~s}$ ). As shown in the figures, the wave-current induced maximum liquefaction areas mostly concentrate on upper layer of seabed foundation where in front of breakwaters, in the gaps between breakwaters and behind breakwaters. It is obvious that liquefaction areas grown with time in all three cases. For example, in the case with one breakwater, the liquefaction areas were quite small at $t=100$ $\mathrm{s}$ and become very large at $t=300 \mathrm{~s}$. This indicates that the wave-current induced liquefaction in elasto-plastic soil is a process of progressive. The effects of number of breakwaters on wave-current induced maximum liquefaction areas can be easily examined by comparing three figures. It can be observed that the liquefaction areas were largest in the case with three breakwaters and smallest in the case with one breakwater at the exact same time. In other words, the number of breakwaters has positive effective on soil liquefaction. This is because that multiple breakwaters can reflect more waves back with Bragg effects which make wave height larger in front of breakwaters. As a result, although the multiple breakwaters can protect coast lines more efficiently, they will also increase the seabed instability (liquefaction) in front 
of them.

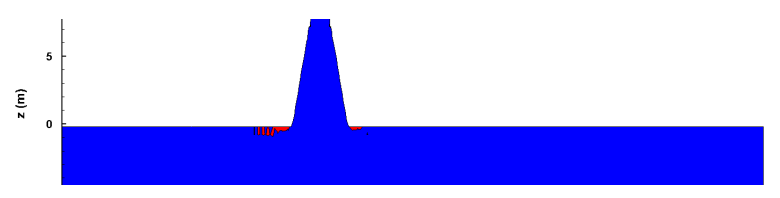

(a) $t=100 \mathrm{~s}$

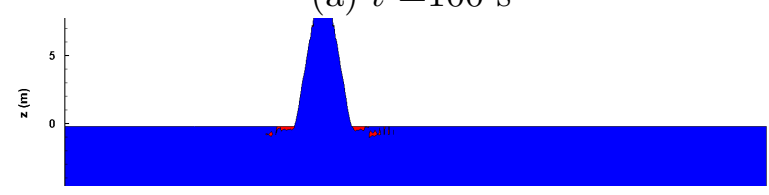

(b) $t=200 \mathrm{~s}$

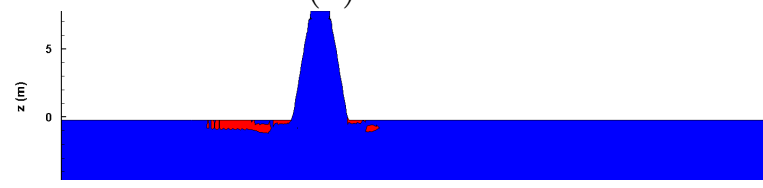

(c) $t=300 \mathrm{~s}$

Fig. 16. Wave-current induced maximum liquefaction area around one breakwater at: (a) $t=100 \mathrm{~s}$; (b) $t=200 \mathrm{~s}$; (c) $t=300 \mathrm{~s}$.

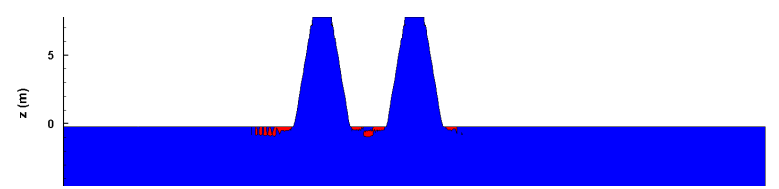

(a) $t=100 \mathrm{~s}$

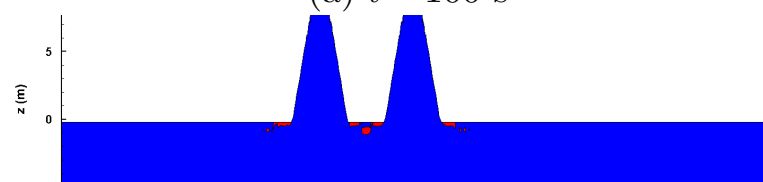

(b) $t=200 \mathrm{~s}$

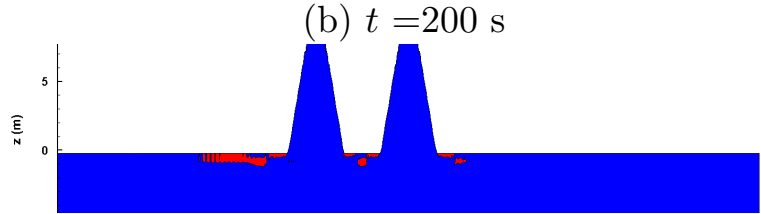

(c) $t=300 \mathrm{~s}$

Fig. 17. Wave-current induced maximum liquefaction area around two breakwaters at: (a) $t=100$ $\mathrm{s}$; (b) $t=200 \mathrm{~s}$; (c) $t=300 \mathrm{~s}$. 

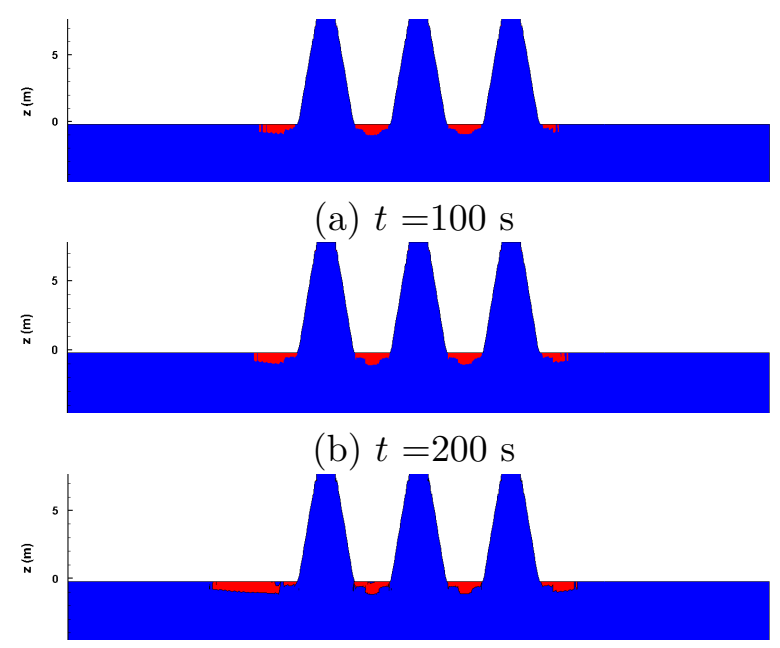

(c) $t=300 \mathrm{~s}$

Fig. 18. Wave-current induced maximum liquefaction area around three breakwaters at: (a) $t=100$ $\mathrm{s}$; (b) $t=200 \mathrm{~s}$; (c) $t=300 \mathrm{~s}$.

\subsection{Parametric study}

In this section, parametric studies including the effects of current, soil properties and wave characteristics on the vertical distribution of liquefaction potential in an elasto-plastic seabed foundation will be discussed. Four typical time are selected to illustrate the progressive soil liquefaction.

Figure 19 demonstrates the vertical distribution of liquefaction potential on $x$ $=-1.5 \mathrm{~m}$ at $t=20 \mathrm{~s}, t=40 \mathrm{~s}, t=150 \mathrm{~s}$ and $t=280 \mathrm{~s}$ for cases with different current conditions (following currents $U_{0}=1 \mathrm{~m} / \mathrm{s}$, no currents $U_{0}=0 \mathrm{~m} / \mathrm{s}$ and opposing currents $\left.U_{0}=-1 \mathrm{~m} / \mathrm{s}\right)$. It is observed that the liquefaction potential in the upper seabed is generally greater than that in the lower seabed. As the wave-current loading keep applying on the seabed foundation, the liquefaction potential at all depth of seabed increases until liquefaction happens. After $L_{p}$ almost reaches 1.0, it would not increase or even decrease a little as the build up pore pressure remains as constant in a nearly liquefied soil. It is shown in the figures that the presence of currents has significant impact on development of liquefaction potential. At $t=20 \mathrm{~s}$ (the early stage), the influence is not obvious, three curves are basically overlapped. As time goes by, the impact becomes more and more obvious. It is clearly that following currents $\left(U_{0}=1 \mathrm{~m} / \mathrm{s}\right)$ have positive effects on the development of soil liquefaction while opposing currents $\left(U_{0}=-1 \mathrm{~m} / \mathrm{s}\right)$ have negative effects compared to the case without presence of currents. In the other words, the following current can deteriorate the seabed foundation instability, while the opposing current can prevent occurrence of soil liquefaction which is good for the offshore structures stability.

Soil properties and wave characteristics may vary at different sites. Therefore, it 

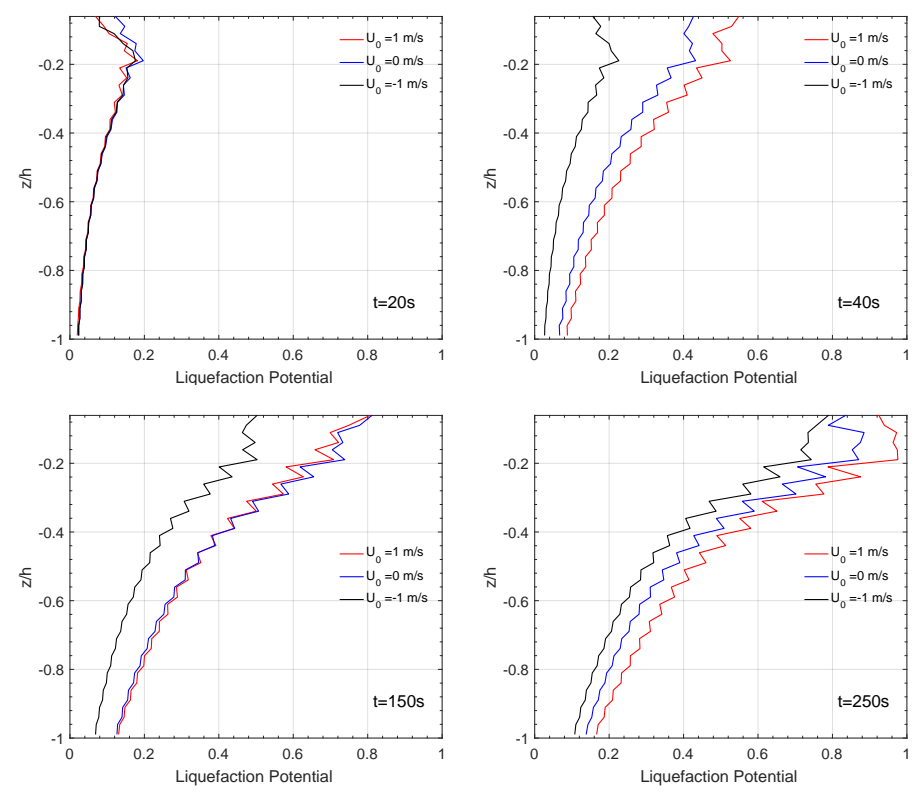

Fig. 19. Effect of currents $U_{0}$ on vertical distribution of liquefaction potential on $x=-1.5 \mathrm{~m}$ at four typical time.

is crucial to examine the effects of soil and wave characteristics on soil liquefaction potential in an elasto-plastic seabed foundation. In this study, soil permeability $K_{s}$, degree of saturation $S_{r}$, wave period $T$, wave height $H_{w}$ and water depth $d$ are considered. Figure 20 shows the effects of soil permeability $K_{s}$ on vertical distribution of wave-current induced liquefaction potential. Similarly, liquefaction potential increases at all depths along with time. It can be observed that the trends of liquefaction potential in soil with $K_{s}=-10^{-7} \mathrm{~m} / \mathrm{s}$ and $K_{s}=-10^{-5} \mathrm{~m} / \mathrm{s}$ are basically identical. While liquefaction potential in soil with $K_{s}=-10^{-2} \mathrm{~m} / \mathrm{s}$ are much smaller (less than 0.2 ), which means the seabed foundation with $K_{s}=-10^{-2} \mathrm{~m} / \mathrm{s}$ will never be liquefied. The main reason of this phenomenon is that the build up and dissipation of pore pressure exist simultaneously in seabed foundation. The soil with large permeability has large pore pressure dissipation rate, which the residual pore pressure could not build up to a high level and the effective stress between soil particles is enough to prevent the soil liquefaction occurs. In conclusion, the soil liquefaction is more likely to happen in a seabed foundation with small soil permeability.

Figure 21 illustrates the effect of degree of saturation $S_{r}$ on vertical distribution of wave-current induced liquefaction potential. As shown in the figure, the soil liquefaction tends to occur in a seabed foundation with a higher degree of saturation. This is due to the compressibility of trapped air in unsaturated soil makes the process of pore pressure build up more difficult. Another interesting phenomenon is that the liquefaction potential decreases significant in the area near seabed surface. This is because the drainage distance is shorter in this area, the pore pressure is 

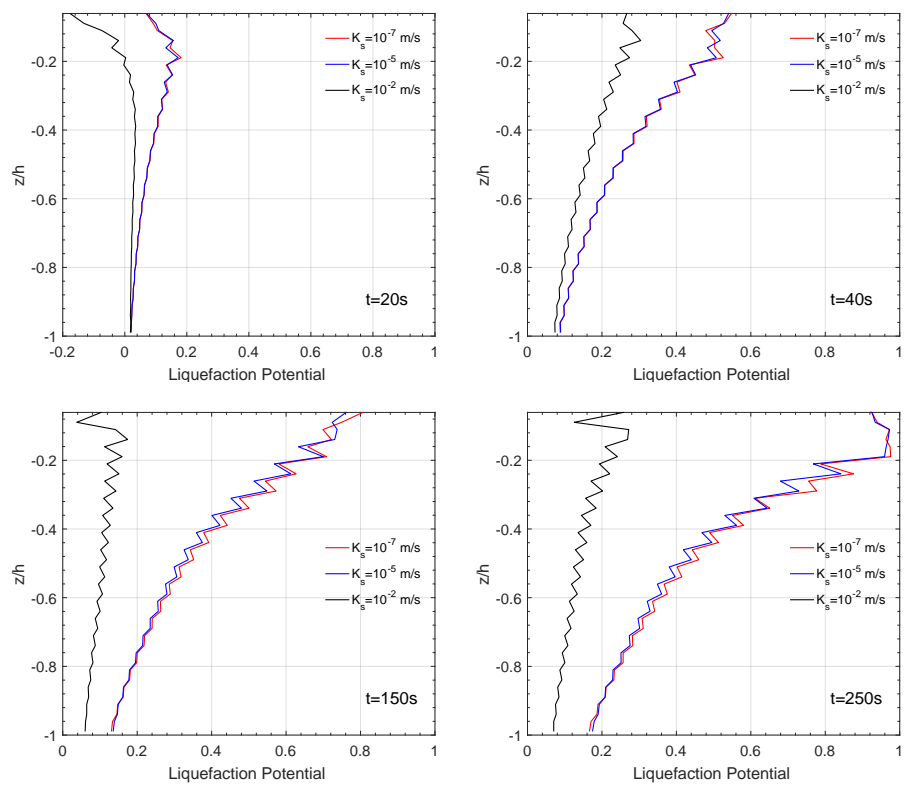

Fig. 20. Effect of soil permeability $K_{s}$ on vertical distribution of liquefaction potential under combined wave and current loading on $x=-1.5 \mathrm{~m}$ at four typical time.

difficult to accumulate and easy to dissipate.
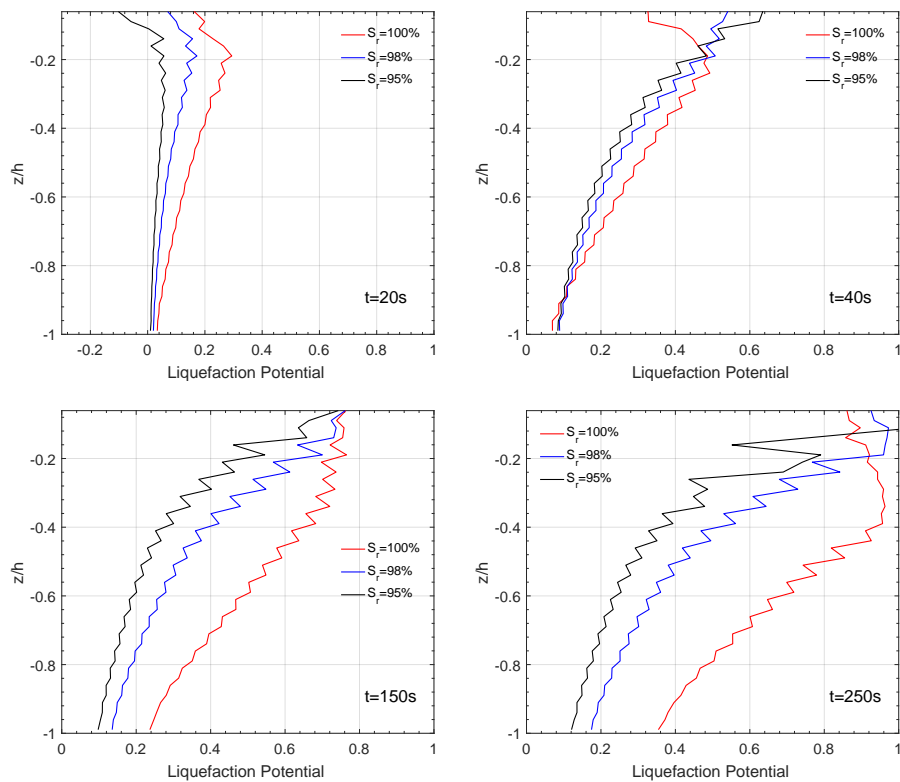

Fig. 21. Effects of degree of saturation $S_{r}$ on vertical distribution of liquefaction potential on $x$ $=-1.5 \mathrm{~m}$ at four typical time. 
Figures 22, 23 and 24 show the effects of wave characteristics (wave period $T$, wave height $H_{w}$ and water depth $d$ ) on wave-current induced liquefaction potential in an elasto-plastic seabed foundation. As seen from these figures, the wave height $\left(H_{w}\right)$ has the most significant effects on the process of liquefaction, while the other two parameters, especially the water depth $(d)$, are not important. In Figure 23, it can be easily observed that larger waves make a seabed foundation more likely to be liquefied. For example, the seabed foundation has been fully liquefied at $t=250$ $\mathrm{s}$ in the case with $H_{w}=5 \mathrm{~m}$. Overall, the vertical distribution of the wave-current induced liquefaction potential in an ealsto-plastic seabed foundation is positively related to wave height $\left(H_{w}\right)$ and wave period $(T)$ and negatively related to water depth $(d)$.
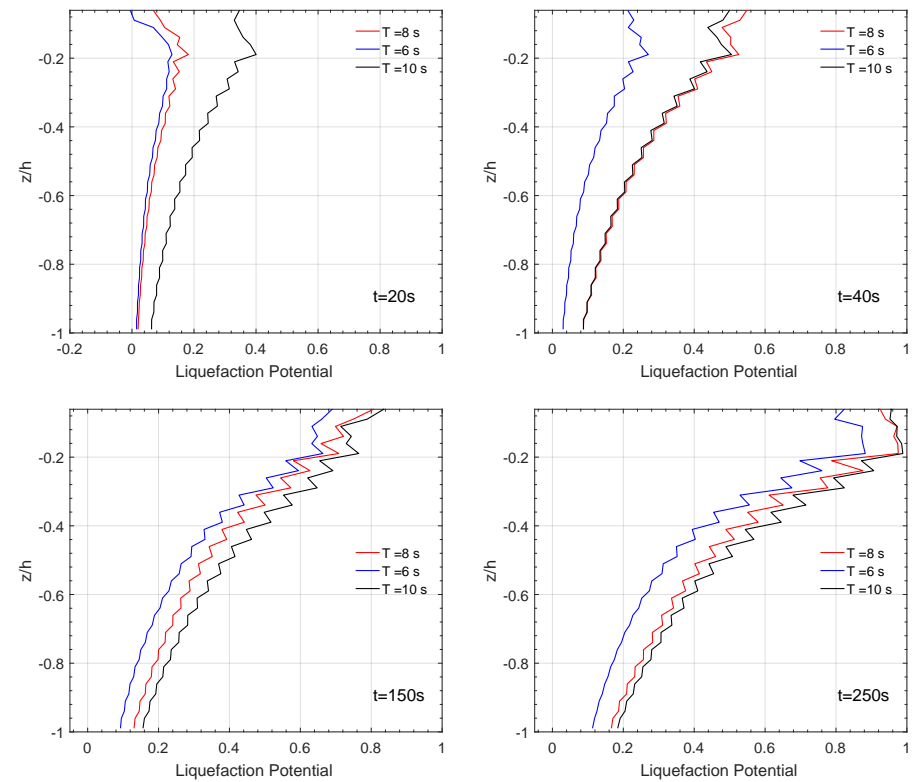

Fig. 22. Effects of wave period $T$ on vertical distribution of liquefaction potential on $x=-1.5 \mathrm{~m}$ at four typical time.

\section{Conclusions}

In this study, an integrated numerical model has been developed to investigate the flow field properties involved in wave-current-breakwaters interactions and corresponding dynamic response in an elasto-plastic seabed foundation around multiple submerged permeable breakwaters with Bragg effects. Based on the numerical results, the following conclusions can be drawn:

(1) The presence of the submerged permeable breakwaters can significantly change the wave pattern and decrease the wave height. The multiple submerged perme- 

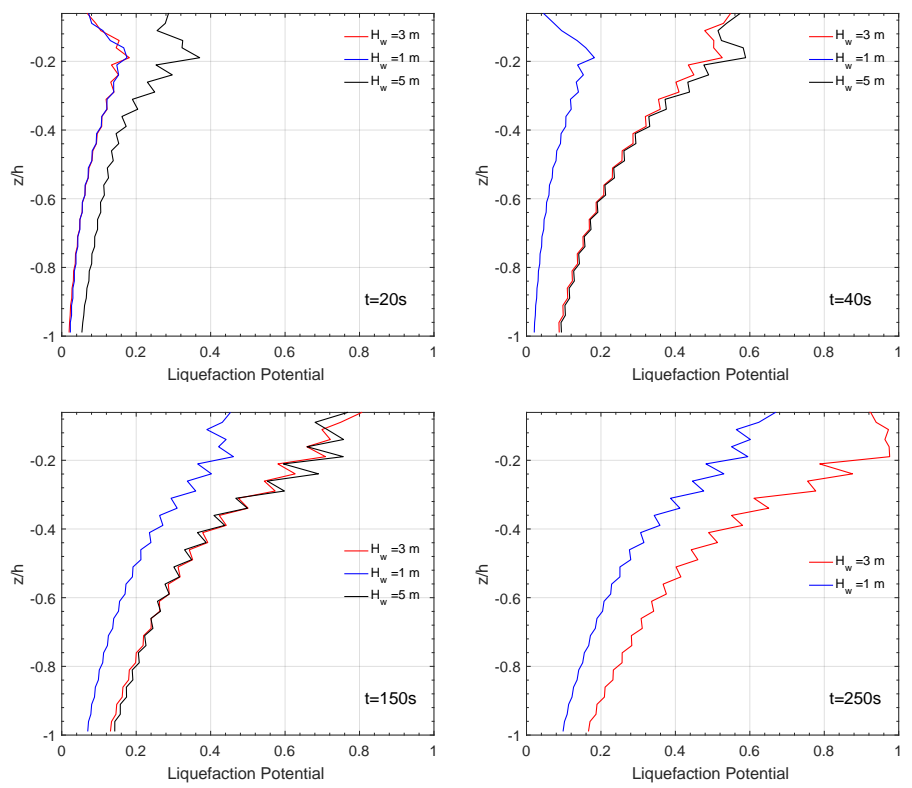

Fig. 23. Effects of wave height $H_{w}$ on vertical distribution of liquefaction potential on $x=-1.5 \mathrm{~m}$ at four typical time.
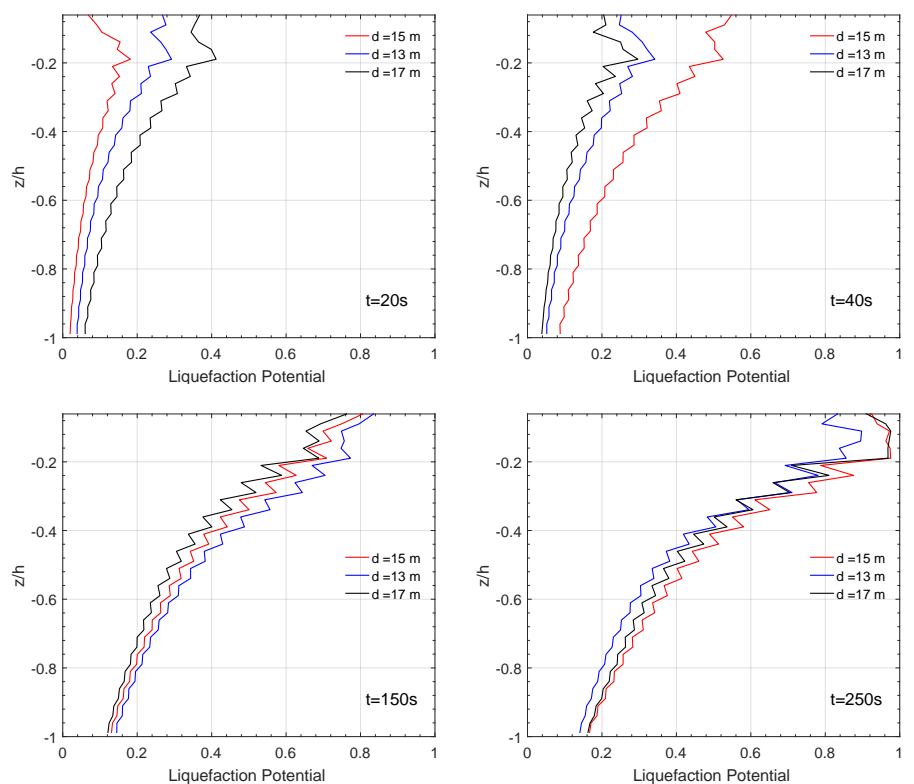

Fig. 24. Effects of water depth $d$ on vertical distribution of liquefaction potential on $x=-1.5 \mathrm{~m}$ at four typical times.

able breakwaters can dissipate the propagating waves more efficiently compared 
to a single breakwater, which provides better protection for the coast line.

(2) Due to the Bragg effect and energy dissipation of submerged permeable breakwaters, the horizontal velocity field behind the breakwaters decreases significantly. The Bragg effect is strongest when the space between two adjacent breakwaters are about half of the wave length. Stronger Bragg effect is better for protecting coastline, however, this may imply more chance for the seabed foundation at seaward side of breakwater to be liquefied.

(3) Construction of breakwaters significantly changes the stress status in seabed foundation. Determination of the stress state after consolidation process completed is essential for the dynamic analysis of seabed foundation and liquefaction potential evaluation.

(4) Under combined wave and current loading, the pore pressure in an elasto-plastic seabed foundation keeps building up to a limited value, accompanying with the reduction of effective stress and shear stress between the soil particles. The presence of the current can significantly affect soil liquefaction potential. The following current aggravates the soil response, which is bad for seabed foundation stability; while the opposing current mitigates the soil response and avoids occurrence of soil liquefaction, which is beneficial for seabed stability.

(5) The loosely deposited seabed foundation has obvious lateral spreading and significant vertical subsidence under combined wave and current loading.

(6) The Bragg effect amplifies the wave height in front of breakwaters and attenuate the wave height behind the breakwaters. Correspondingly, the build up of pore pressure and reduction of effective stress are more significant in front of breakwaters compared to those behind the breakwaters. In the zone beneath three breakwaters or between breakwaters, due to compression of breakwaters, the initial effective stresses are large and more difficult to decrease to zero.

(7) The liquefaction potential in an elasto-plastic seabed foundation increases over time and decreases with the increasing of depth. This indicates that the liquefaction is more likely to happen in the upper part of seabed foundation.

(8) The parametric study shows that the presence of currents has significant impact on development of liquefaction potential in seabed foundation. The following currents can deteriorate the seabed foundation instability, while the opposing currents can prevent occurrence of soil liquefaction which is good for offshore structures stability. Parametric studies also show that wave-current induced liquefaction is more likely to happen in seabed foundation with small soil permeability or fully-saturated. Larger wave height or longer wave period also increase soil liquefaction potential.

In this study, the effects of the currents and interactions with structures (i.e., submerged breakwaters) on the soil responses are considered. Although the investigations on the current effects might be preliminary, it shows the significant impacts on the soil response. 


\section{Acknowledgements}

The authors gratefully acknowledge the support of the Griffith University Research Service Team and the use of the High Performance Computing Cluster Gowonda to complete this research. The first author is thankful for the support of the Griffith University International Postgraduate Research Scholarship and the Griffith University Postgraduate Research Scholarship.

\section{Appendix: Sensitivity analysis of $E_{1}$ and $E_{2}$}

In general, $E_{1}$ and $E_{2}$ in (3) should be calibrated from the physical tests, however, the experimental values of $E_{1}$ and $E_{2}$ for present simulations are not available, the only option is to perform a sensitivity analysis of the parameters on the degree of changes of the solutions regarding to the friction parameters.

The sensitivity analysis of the present simulation conditions have been done, and the numerical results show that the hydrodynamic pressure at locations both inside and outside of breakwaters is not sensitive to the variation of the friction factors, as shown in Fig. 25. Furthermore, the velocity and free surface elevation changes with different friction factors are also checked and found that the $E_{2}$ values have a huge influence on the velocity, $E_{1}$ values have very little impact on velocity (see Fig. 26). For the free surface elevation, the results remain identical, as shown in Fig. 27. Since only the hydrodynamic pressure is used from the flow sub-model to apply to the soil sub-model (one-way coupling algorithm), which means for present material and flow conditions, the parameter $E_{1}$ and $E Z_{2}$ barely have an influence on the simulation results. Therefore, the default values are used in this study.
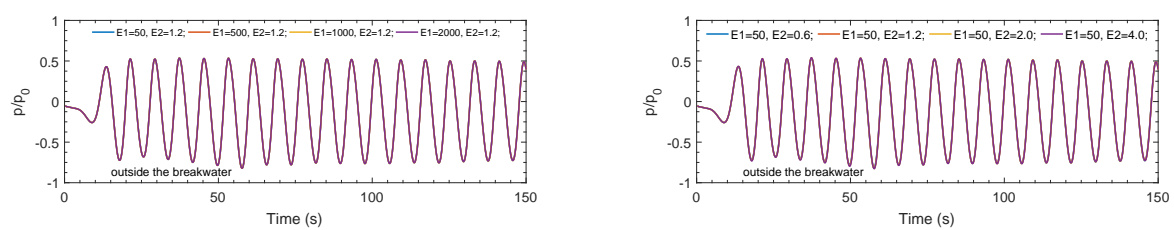

(a) Outside the breakwaters
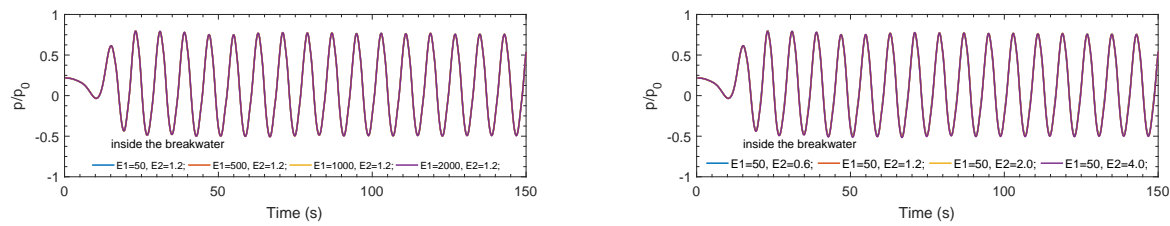

(a) Inside the breakwaters

Fig. 25. Sensitivity analysis of $E_{1}$ and $E_{2}$ for dynamic wave pressures. 

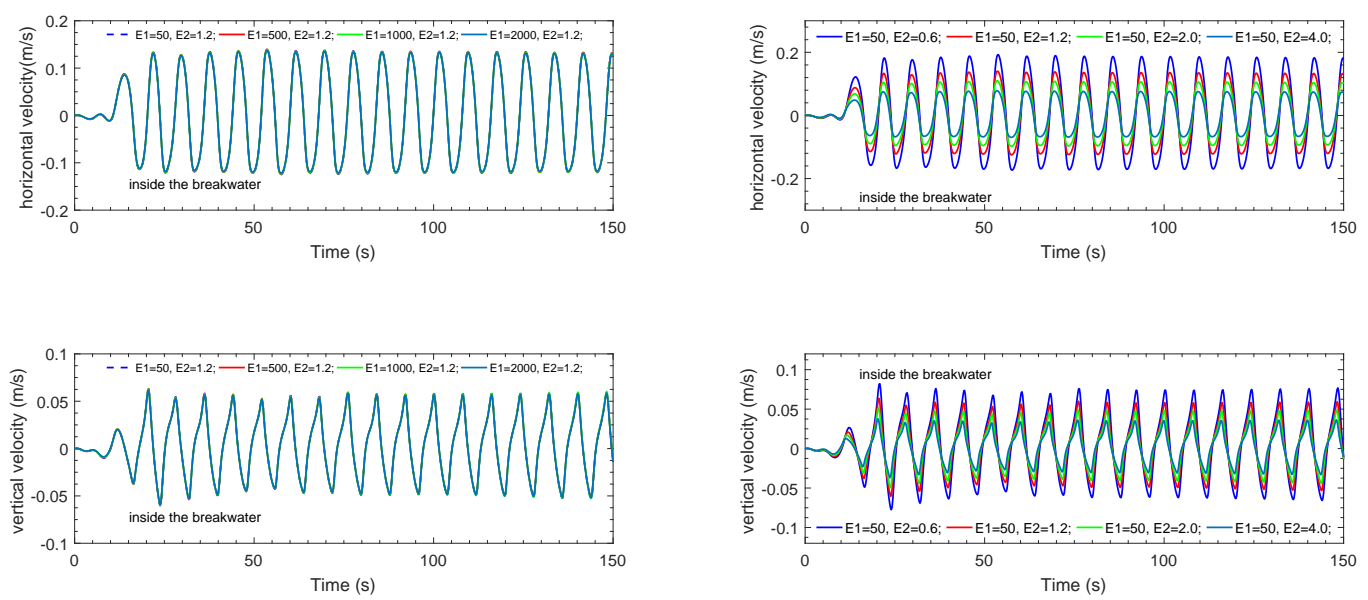

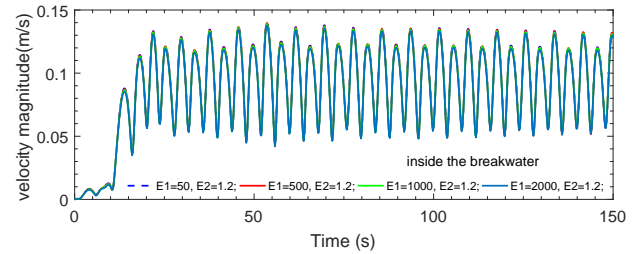

(a) Effect of $E_{1}$

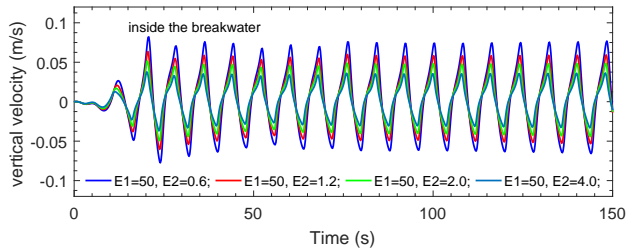

(b) Effect of $E_{2}$

Fig. 26. Sensitivity analysis of $E_{1}$ and $E_{2}$ for the velocity.

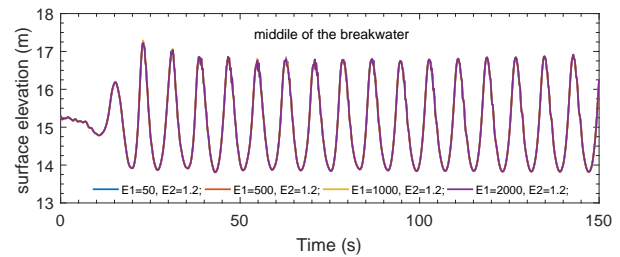

(a) Effect of $E_{1}$

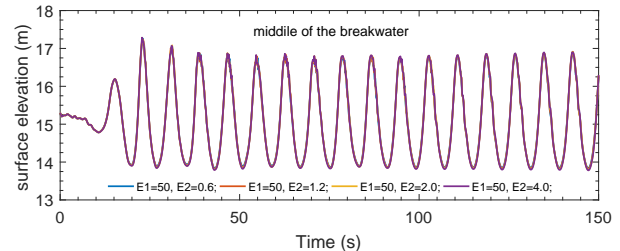

(b) Effect of $E_{2}$

Fig. 27. Sensitivity analysis of $E_{1}$ and $E_{2}$ for the free surface elevation.

\section{References}

Burcharth, H. F. \& Andersen, O. K. [1995] On the one-dimensional steady and unsteady porous flow equations, Coastal Engineering 24(3-4): 233-257.

Chan, A. H. C. [1998] A unified finite element solution to static and dynamic problems of geomechanics, Ph.D. Thesis, University College of Swansea, UK.

Cho, Y. S., Lee, J. I., \& Kim, Y. T. [2004] Experimental study of strong reflection of regular water waves over submerged breakwaters in tandem, Ocean Engineering 31(10): 1325-1335.

Dunn, S. L., Vun, P. L., Chan, A. H. C. \& Damgaard, J. S. [2006] Numerical modeling of wave- 
induced liquefaction around pipelines, Journal of Waterway, Port, Coastal, and Ocean Engineering, ASCE 132(4): 276-288.

Engelund, F. [1953] On the laminar and turbulent flows of ground water through homogeneous sand, Akad, for de Tekniske Videnskaber.

Higuera, P., Lara, J. L. \& Losada, I. J. [2013] Realistic wave generation and active wave absorption for navier-stokes models: Application to openfoam $囚$, Coastal Engineering, 71: 102-118.

Higuera, P. [2015] Application of computational fluid dynamics to wave action on structures, $\mathrm{Ph} . \mathrm{D}$. Thesis, Universidade de Cantabria.

Hsu, T. J., Sakakiyama, T. \& Liu, P. L. F. [2002] A numerical model for wave motions and turbulence flows in front of a composite breakwater, Coastal Engineering 46(1): 25-50.

Jeng, D.-S. [1996] Wave-induced liquefaction potential at the tip of a breakwater: an analytical solution, Applied Ocean Research 18(5): 229-241.

Jeng, D.-S. [2003] Wave-induced sea floor dynamics, Applied Mechanics Reviews 56(4): 407-429.

Jeng, D.-S., Schacht C. \& Lemckert C. [2005] Experimental study on ocean waves propagating over a submerged breakwater in front of a2240.

Jeng, D.-S. \& Ou, J. [2010] 3D models for wave-induced pore pressures near breakwater heads, Acta Mechanica 215(1-4): 85-104.

Jeng, D.-S. [2013] Porous Models for Wave-seabed Interactions, Springer, Heidelberg, 289pp.

Jeng, D.-S. [2018] Mechanics of Wave-Seabed-Structure Interactions: Modelling, Processes and Applications, Cambridge University Press, Cambridge, 358pp.

Jensen, B., Jacobsen, N. G., \& Christensen, E. D. [2014] Investigations on the porous media equations and resistance coefficients for coastal structures, Coastal Engineering 84: 56-72.

del Jesus, M. [2011] Three-dimensional interaction of water waves with maritime structures, Ph.D. Thesis, University of Cantabria.

Jiaer, W., Kammerer, A., Riemer, M., Seed, R. \& Pestana, J. [2004] Laboratory study of liquefaction triggering criteria, 13th World Conference on Earthquake Engineering, Vancouver, BC, Canada, 2580 .

Lan, Y. J., Hsu, T. W., Lai, J. W., Chang, C. C., \& Ting, C. H. [2011] Bragg scattering of waves propagating over a series of poro-elastic submerged breakwaters, Wave Motion48(1): 1-12.

Lin, P. \& Liu, P. L. F. [1998] A numerical study of breaking waves in the surf zone, Journal of Fluid Mechanics 359: 239-264.

Liu, P. L. F., Lin, P., Chang, K. A. \& Sakakiyama, T. [1999] Numerical modelling of wave interaction with porous structures, Journal of Waterway, Port, Coastal, and Ocean Engineering, ASCE, 125(6): 322-330.

Liu, Z., Jeng, D.-S., Chan, A. H. C. \& Luan, M. [2009] Waveinduced progressive liquefaction in a poroelastoplastic seabed: A twolayered model, International Journal for Numerical and Analytical Methods in Geomechanics 33(5): 591-610.

Mase, H., Kimura, A., \& Sakakibara, H. [1996] Resonant reflection and refraction-diffraction of surface waves due to porous submerged breakwaters, Proceedings of 25th Conference on Coastal Engineering, (ICCE95), Orlando, Florida, Chapter 183: 2366-2376.

Mei, C. C., Stiassnie, M. \& Yue, D. K. P. [2005] Theory and Applications of Ocean Surface Waves: Nonlinear Aspects. World scientific.

Mizutani, N., Mostafa, A. M. \& Iwata, K. [1988] Nonlinear regular wave, submerged breakwater and seabed dynamic interaction, Coastal Engineering, 33(2): 177-202.

Mostafa, A. M., Mizutani, N. \& Iwata, K. [1999] Nonlinear wave, composite breakwater, and seabed dynamic interaction, Journal of Waterway, Port, Coastal, and Ocean Engineering, ASCE, 125(2): 88-97.

Okusa, S. [1985] Wave-induced stresses in unsaturated submarine sediments, Géotechnique 35(4): $517-532$.

Pastor, M., Zienkiewicz, O. \& Chan, A. H. C. [1980] Generalized plasticity and the modelling of soil behaviour, International Journal for Numerical and Analytical Methods in Geomechanics 14(3): 151-190.

Sassa, S., \& Sekiguchi, H. [1999] Wave-induced liquefaction of beds of sand in a centrifuge, 
Géotechnique 49(5): 621-638.

Sassa, S., \& Sekiguchi, H. [2001] Analysis of wave-induced liquefaction of sand beds, Géotechnique 51(2): 115-126.

Sassa, S., Sekiguchi, H. \& Miyamoto, J. [2001] Analysis of progressive liquefaction as a movingboundary problem, Géotechnique 51(10): 847-857.

Sumer, B. M. and Fredsøe J. [2002] The mechanics of scour in the marine environment, World Scientific, New Jersey.

Sumer, B. M., Hatipoglu, F., Fredsøe, J. \& Kaan Sumer, S. [2006] The sequence of sediment behaviour during waveinduced liquefaction, Sedimentology 53(3): 611-629.

Sumer, B. M. [2009] Liquefaction around marine structures, Coastal Structures 2007, 1864-1870.

Sumer, B. M., Ozgur Kirca, V. S., \& Fredsøe, J. [2012] Experimental validation of a mathematical model for seabed liquefaction under waves, International Journal of Offshore and Polar Engineering 22(2): 133-141.

Sumer, B. M. [2013] Advances in seabed liquefaction and its implications for marine structures, Geotechnical Engineering 45(4): 1-14.

Sumer, B. M. [2014] Liquefaction around marine structures, World Scientific, New Jersey.

Umeyama, M. [2010] Coupled PIV and PTV measurements of particle velocities and trajectories for surface waves following a steady current, Journal of Waterway, Port, Coastal, and Ocean Engineering, ASCE 137(2): 85-94.

Yamamoto, T., Konning, H., Sellmeijer, H. \& Hijum, E. V. [1978] On the response of a poro-elastic bed to water waves, Journal of Fluid Mechanics 87(1): 193-206.

Ye, J. H. \& Jeng, D.-S. [2012] Response of porous seabed to nature loadings: waves and currents, Journal of Engineering Mechanics, ASCE 138(6): 601-613.

Ye, J. [2012] Numerical analysis of wave-seabed-breakwater interactions, Ph.D. Thesis, University of Dundee.

Ye, J., Jeng, D.-S., Wang, R. \& Zhu, C. [2013] Validation of a 2-d semi-coupled numerical model for fluid-structure-seabed interaction, Journal of Fluids and Structures 42: 333-357.

Ye, J., Jeng, D.-S., Wang, R. \& Zhu, C. [2015] Numerical simulation of the wave-induced dynamic response of poro-elastoplastic seabed foundations and a composite breakwater, Applied Mathematical Modelling 39(1): 322-347.

Zhang, J. S., Jeng, D.-S., Liu, P. F., Zhang, C. \& Zhang, Y. [2012] Response of a porous seabed to water waves over permeable submerged breakwaters with bragg reflection, Ocean Engineering 43: $1-12$.

Zienkiewicz, O. C., Chang, C. \& Bettess, P. [1980] Drained, undrained, consolidating and dynamic behaviour assumptions in soils, Géotechnique, 30(4): 385-395.

Zienkiewicz, O. C. \& Mroz, Z. [1984] Generalized plasticity formulation and applications to geomechanics, Mechanics of Engineering Materials 44(3), 655-680.

Zienkiewicz, O. C., Chan, A. H. C., Pastor, M., Schrefler, B., \& Shiomi, T. [1999] Computational Geomechanics. Springer. 